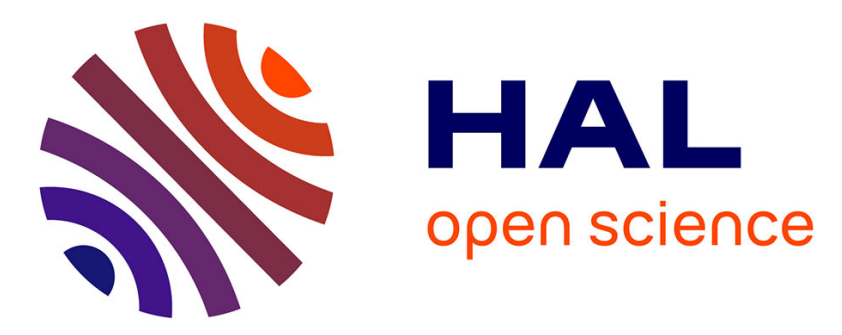

\title{
Understanding Occupant Behaviors in Dynamic Environments using OBiDE framework
}

\author{
Muhammad Arslan, Christophe Cruz, Dominique Ginhac
}

\section{To cite this version:}

Muhammad Arslan, Christophe Cruz, Dominique Ginhac. Understanding Occupant Behaviors in Dynamic Environments using OBiDE framework. Building and Environment, 2019, 166, pp.106412. 10.1016/j.buildenv.2019.106412 . hal-02354572

\section{HAL Id: hal-02354572 \\ https://u-bourgogne.hal.science/hal-02354572}

Submitted on 27 Feb 2021

HAL is a multi-disciplinary open access archive for the deposit and dissemination of scientific research documents, whether they are published or not. The documents may come from teaching and research institutions in France or abroad, or from public or private research centers.
L'archive ouverte pluridisciplinaire HAL, est destinée au dépôt et à la diffusion de documents scientifiques de niveau recherche, publiés ou non, émanant des établissements d'enseignement et de recherche français ou étrangers, des laboratoires publics ou privés. 


\title{
Understanding Occupant Behaviors in Dynamic Environments using OBiDE framework
}

\author{
Muhammad Arslan ${ }^{\mathrm{a}, *}$, Christophe $\mathrm{Cruz}^{\mathrm{a}}$, Dominique Ginhac ${ }^{\mathrm{b}}$ \\ ${ }^{a}$ Laboratoire d'Informatique de Bourgogne (LIB), Univ. Bourgogne Franche-Comte, EA 7534, Dijon, France \\ ${ }^{\mathrm{b}}$ Laboratoire Imagerie et Vision Artificielle (ImViA), Univ. Bourgogne Franche-Comte, EA 7535, Dijon, France
}

\begin{abstract}
A B S T R A C T
Occupants' movements and presence are fundamental and the pre-requisites for any type of occupant behaviors' understanding which tells whether a building location is occupied, the number of occupants or an occupant with a specific profile in a certain location. Numerous studies have been conducted over the past few decades to model occupant behaviors stochastically for an improved understanding of their activities for different facility management applications. Despite many research efforts to model dynamic behaviors of building occupants, the understanding of their behaviors by incorporating the contextual information linked to the evolution of the building environment is still not adequately explored. The contextual information linked to locations in dynamic environments changes often over time in terms of position, size, properties and relationships with the en-vironment. This changing building environment affects the occupants' movements and presence inside the fa-cility which ultimately degrades the process of inferring their accurate activities based on the location context. Henceforth, the evolving building information is required to be mapped with occupant movements for an im-proved understanding of their changing behaviors. To fill this research gap, a framework named 'Occupant Behaviors in Dynamic Environments' (OBiDE) is designed for providing a 'blueprint map' to integrate existing DNAS (Drivers, Needs, Systems, Actions) ontology (i.e. a scheme to model occupant behaviors) with our se-mantic trajectory enrichment model to better understand the occupant behaviors by tracking the dynamicity of building locations. The proposed framework extends the usability of DNAS by providing a centralized knowledge base that holds the movements of occupants with relevant historicized contextual information of the building environment to study occupant behaviors for different facility management applications.
\end{abstract}

\section{Introduction}

Occupants are the important factor for building monitoring and management operations as they impact the building environments in terms of heat production as well as through energy consumption by operating the building appliances [1]. For ensuring an appropriate level of quality of services to the building occupants, the most crucial challenge faced by the facility managers is to understand the occupant behaviors and their interactions with buildings [1,2]. Although, this is a complex activity because the occupant behaviors and the buildings are dynamic in nature and context-dependent. Here, a context refers to any information based on the contextual factors such as space, time and environment utilized for categorizing the situation of occupants [2,3]. Failure in understanding occupant behaviors because of inadequate integration of all relevant contextual factors associated with the occupants and the building environments can result in serious economic and administrative crises such as under-utilization of the building spaces, decreased efficiency of occupants due to poor environmental conditions, increased energy usage, and safety-related hazards [1-4]. On the contrary, if the occupant behaviors are modeled effectively by including all the possible contextual factors (functionality of the building locations, social-personal, economic, etc.) which may affect occupant behaviors will lead to an enhanced physical comfort, increased safety at work and improved work performance of the occupants while keeping the level of building resources to the optimum $[1,5]$. Existing literature [4-9] encompasses many studies for constructing systems for modeling occupant behaviors which help facility managers in decision making for building operations by stochastically modeling the dynamic behaviors of occupants after incorporating the random variations in their behaviors over time. Despite such numerous existing studies, the development and application of such systems are missing in the literature which can track the changes occurring in a building during its lifecycle

\footnotetext{
* Corresponding author.

E-mail addresses: muhammad.arslan@u-bourgogne.fr (M. Arslan), christophe.cruz@ubfc.fr (C. Cruz), dominique.ginhac@ubfc.fr (D. Ginhac).
} 


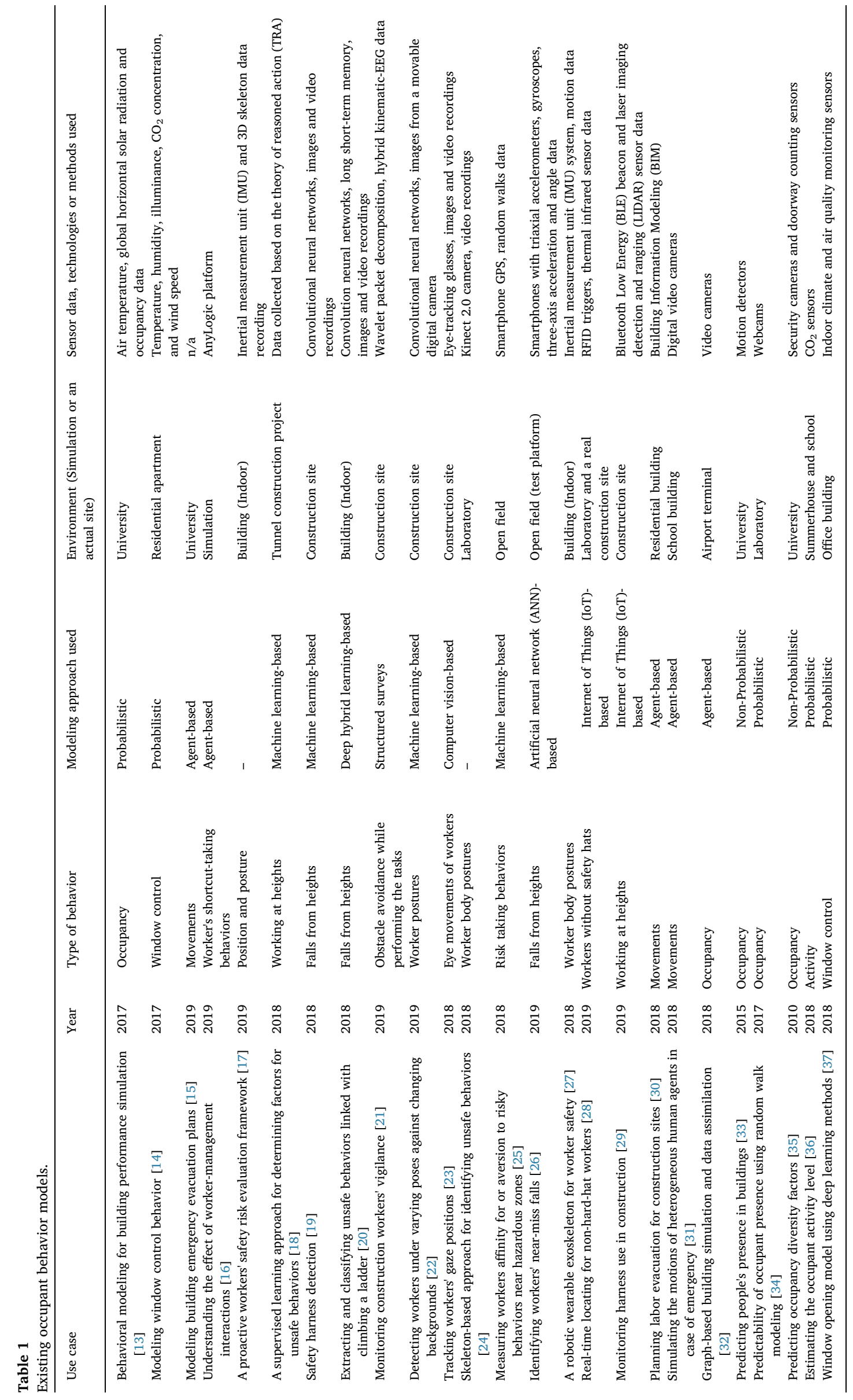


and can be used to study occupant behaviors (movements and their interactions with the building) using the evolving contextual information of the building environment. To fill this research gap, initially existing occupant behavior systems were reviewed for defining the occupant behaviors which resulted in choosing a DNAS ontology [4] based on its relevance to our case-study and application as well as its acceptance that is perceived from its citations. A DNAS ontology has four components which are; 1 . the 'drivers', 2. the 'needs', 3. the 'actions' which building occupants perform to fulfill their needs and 4. the 'systems' through which the occupants interact with a building for performing actions to fulfill their needs. Although, the application of a DNAS ontology [4] is primarily explored for energy-related occupant behavior modeling in the existing literature. However, this ontology can be treated as a foundation to represent other types of occupant behaviors in buildings as it provides a basic structure to represent behaviors using four core components as discussed above. For instance, in our proposed framework (i.e. OBiDE), a DNAS ontology is used for defining the safety-related occupant behaviors. For understanding the occupant movements (the pre-requisite for any kind of behavioral understanding) with the evolving building context [10], the dynamicity of a building environment in terms of geometrical and contextual information is achieved using our 'Semantic Trajectories in Dynamic Environments' (STriDE) model $[3,11]$. The STriDE is a semantic enrichment data model based on ontologies which aims to provide a centralized knowledge base that holds the spatio-temporal movements of occupants with relevant historicized contextual and geometrical information of the building infrastructure [11]. For an improved understanding of occupant behaviors by decoding and classifying their different types of movements, the stored occupant spatio-temporal data is later fed to a probabilistic model (i.e. Hidden Markov Model) for inferring their movement states [12]. The computation of probabilities of different movements of occupants in terms of evolving building locations is used for enriching a DNAS ontology for an enhanced understanding of occupant behaviors by categorizing their actions based on their movements for our safety management application. The casestudy dealing with the occupants' safety management scenario is purely to show a proof-of-concept application of our OBiDE framework. However, the proposed framework can also be used for different facility management scenarios where there is a need to fuse occupants' movements and presence data with the evolving geometrical and contextual information of buildings.

The rest of the paper is organized as follows: Section 2 describes the background of the study. First, it defines the occupant behaviors. Second, it describes the main steps involved in occupant behavior modeling. Third, existing occupancy detection techniques are explored. Lastly, the importance of fusing the dynamicity of building environments with building occupancy information is stated. Section 3 is based on the proposed integrated framework. Section 4 presents a brief casestudy using the proposed framework. Section 5 presents a discussion and Section 6 describes a conclusion with some future works.

\section{Background}

\subsection{What are occupant behaviors?}

Behaviors are observable actions or reactions of a user in response to external or internal stimuli [1]. These actions or reactions can be categorized into four main types which are; physiological adjustments (e.g. sweating, shivering, etc.), individual adjustments (e.g. selection of clothes, using earplugs, etc.), environmental adjustments (HVAC adjustment, window opening or closing, etc.) and spatial adjustments (moving from one building facility to another, etc.) [1]. In other words, behaviors are the interactions (leaving or entering a room, visual and thermal indoor conditions adjustment using windows or blinds, doors, etc.) of building occupants which can be categorized into different movements, simple presence or actions with their environment (building, its systems and appliances) which impact on the building performance (heating or cooling, indoor air quality, energy, comfort, etc.) during their stay in a building [2]. Thereby, an occupant interaction which results in changing a building state (presence or absence in case of occupancy monitoring) or no interaction leaving the present state of a building unchanged are both facets of occupant behaviors [1]. Existing literature contains numerous systems to model occupant behaviors shown in Table 1. For modeling the occupant behaviors and their interactions with the building, the occupants' movements and presence are the preconditions for any kind of behavior understanding as building occupants can only interact with the building environment if they are present inside the building [5,11].

\subsection{Occupant behavior modeling}

Primarily, there exist four major types of approaches to model occupant behaviors which are: static-deterministic, static-stochastic, dynamic-deterministic and dynamic-stochastic $[1,2,4,5]$. The static models do not have the ability to capture the influences that a building and its occupants can have on each other [5]. These models are designed for understanding non-adaptive behaviors e.g. turning off the lights when occupants are going for a holiday, etc. Conversely, dynamic models deal with two-way interactions between a building and its occupants and are suitable for adaptive nature of occupants' behaviors e.g. turning on the lights, changing the heating or cooling of a building, etc. Deterministic models produce the same outcomes every time when a simulation is run and give the homogeneous and deterministic results. Whereas, stochastic models produce different output every time when a simulation is run because the modeling parameters are selected randomly [5]. Among all the approaches discussed above, the modeling approach which has been used extensively in the industry is static-deterministic modeling [1,5]. However, this modeling approach is not suitable for constructing a robust building design as the uncertainty of occupants' behaviors is not considered. To include the uncertainty of the building occupants' behaviors, stochastic models are recommended [5].

The three types of most commonly used stochastic models are; (1) Markov chain models, (2) Bernoulli models and (3) survival models [5]. Discrete-time and discrete-event are the two main types of Markov chain models which take into an account the environmental conditions for predicting occupants' actions in the latest timestep or an event [5]. The major limitation of Markov chain models is that they are not feasible to apply to the entire population of occupants as the computation and modeling effort increases linearly as the number of occupants in a building increases. In contrast to Markov chain models, Bernoulli models are the most simplified memoryless stochastic models in which the probabilities of events are independent of the previous events [5]. Hence, Bernoulli processes do not require much information for modeling occupants' behaviors. Bernoulli modeling is used for energy modeling at a large scale as its scope can be efficiently applied to the entire building [5]. However, Bernoulli processes do not output individual occupant behavior and are not capable of predicting the timings of individual occupant's behaviors. The third type of modeling approach i.e. survival modeling is used for estimating the time duration until an event occurs in a building. For example, these models are used for estimating how long a building probably remains unchanged by its occupants [5]. In addition to three basic types of modeling approaches as discussed above, there exists an extension of the Markov chain models which use agent-based modeling. Agent-based models predict the influence of occupants by modeling individuals, their mutual interactions and how they interact with their building environment [5]. In agent-based modeling, a huge amount of information (i.e. defining role and relationships between the agents) is typically required and thus increases the modeling complexity. The term complexity is defined as the number of details required for modeling which is dependent on size (number of model components) and a resolution (number of model 
variables) [1,5]. An agent's description generally consists of their attributes, resources, behavioral rules, etc. An extensive range of human agents present in the literature which include agents subject to reinforcement or belief-based learning, non-adaptive agents, and agents with capabilities of evolving new behaviors [5].

After selecting the most appropriate behavior modeling approach as per the application requirements, to create the understanding of the occupant interactions, the modeling process conventionally initiates from the sensor data acquisition of occupants [1,5] along with the building environmental or infrastructural parameters. The methods for collecting occupant behaviors can be divided into three main categories, which are; 1) physical acquisition which can be a) on-site data collection or laboratory studies in controlled environments, 2) surveys, interviews and focus groups, and 3) virtual reality experiments [5]. Physical acquisition studies involve monitoring building occupants in their physical environment. This environment can be an actual site where the occupants are present or a pre-fabricated environment in a laboratory setting that is controlled for a specific time to investigate the occupant behaviors. However, laboratory studies are expensive to build and may infer the behaviors differently as in real buildings the stress level of the occupants is higher [5]. Moreover, the visibility of monitoring sensors installed in laboratories makes occupants conscious that they are been monitored which ultimately constrains their behaviors [5]. Surveys and focus groups depend on the self-reporting of individual behaviors by filling out the questionnaires or through interviews. This method is cost-effective and enables to acquire behaviors which are not possible to measure using sensors (for instance; perception, attitudes, etc.). However, existing studies [5] show that reported behaviors of building occupants may not always correlate with their actual behaviors. In addition, misunderstanding of the questions in the surveys may also lead to incorrect reporting the information about the occupants [5]. Apart from these two traditional approaches, the most emerging approach for occupant behavior modeling is virtual reality-based environments. This approach provides greater control of the environment in terms of environmental conditions (e.g. building layouts). However, there is limited support for visual and air quality configurations [1].

An extensive range of different types of sensors (wired and wireless) for monitoring occupants and the environment are present in the literature [1-5] to acquire information for modeling occupant behaviors and their interactions (energy consumption, etc.) with the buildings. Some of them are mentioned below.

- Mechanical sensing such as door badges, piezoelectric mats, etc.

- Image-based sensing using visual information captured from the cameras.

- Motion-based sensing such as passive infrared (PIR), ultrasonic Doppler and ultrasonic ranging sensors

- Manual observations need humans for collecting datasets

- Wireless RF sensing using ambient sensors for temperature, humidity, light, etc.

- Consumption sensing for measuring water, electricity and gas usage in buildings

For selecting a sensor technology for data acquisition, there exists nine factors $[1,5]$ which needs to be considered which are; 1 . cost (including acquiring, installing and operating cost of the sensors), 2. power type (battery or main powered), 3. accuracy (difference between sensed data values and ground truth), 4. sensor coverage range (distance and a view angle the sensors cover), 5. data collection type (event-based or periodic), 6. data storage (onboard storage of sensors), 7. deployment region (building inside or outside), 8. deployment level and 9. data sensed (binary data or value-based sensing). The quality of data captured from various types of sensors differ greatly in terms of the resolution of the deployed sensors (see Fig. 2). The spatial, temporal and occupant resolutions are combined for determining the overall resolution of the system for capturing the occupant behaviors [1,5]. The

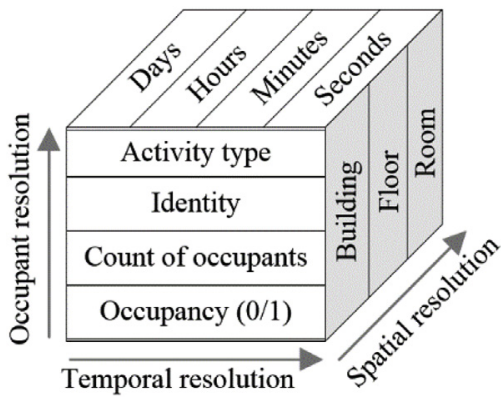

Fig. 1. Occupancy resolution $[1,5]$.

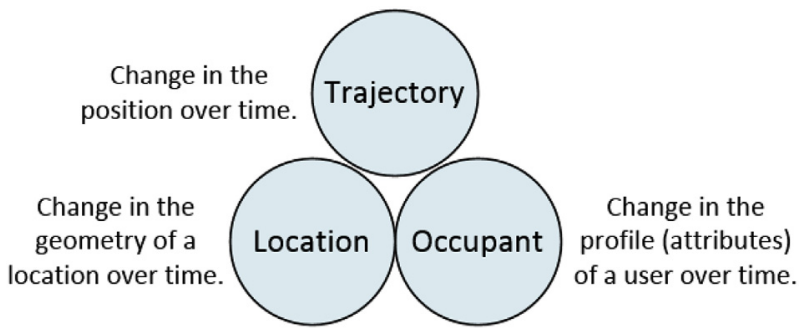

Fig. 2. Entities of a dynamic building environment.

spatial resolution is defined in terms of building infrastructure (i.e. floor, rooms). Whereas occupant resolution can have 4 different levels of information which are; 1) simple detection based on the occupant presence in 0 or 1 values, 2) counting the number of occupants, 3) identifying the occupants, and 4) recognizing the occupants' activities. Along with spatial and occupant resolution, temporal resolution defines the smallest time period in which variations in spatial and occupant resolutions can be informed by a deployed sensor. As the resolution of the captured sensor data increases, the building space gets smaller, the occupants become more distinct based on their identities and the information from the sensor data will be accessed faster [1]. For example, a low-resolution system will only capture the binary information (presence or absence) of the occupants in a specific time where the identities of the occupants are not recognized. Whereas, a high-resolution system will be able to detect the number of occupants, their identifications, as well as their activities.

\subsection{Occupancy detection techniques}

While the previous section provides a brief overview of different types of sensors available for capturing different occupant behaviors, but occupants' movements and presence are the pre-requisites for any type of occupant behaviors' understanding. Existing studies on occupancy detection and proximity analysis have been dominated by a wide range of sensors including passive infrared, Radio-Frequency Identification (RFID), ultrasonic, acoustic recognition, image cameras, Wi-Fi-based, Global Positioning System (GPS) and Bluetooth Low Energy (BLE) technology [38]. Each technology has its own advantages and disadvantages which can be found in Huang et al. [38] research. The output of a passive infrared sensor is binary and used for detecting the occupant presence instead of calculating the precise number of occupants in a facility [39]. RFID-based sensors are conventionally used for calculating course-grained locations and occupancy [40]. Though, as each RFID tag is mapped with an occupant, the privacy of an occupant is the main concern while using this technology [38]. Occupancy detection using ultrasonic technique presents several disadvantages such as complexity in sensor configuration and management [41]. Audio-based occupancy monitoring is inexpensive as the main equipment that is required consists of only microphones with microcontrollers. However, acoustic-based sensors are seldom used for 
occupancy monitoring as non-human sourced sound waves originating inside the noisy buildings can generate many errors in the collected occupancy data [38]. Image cameras are also used for monitoring the building occupancy and estimating their locations in a building [42]. However, the restrictions in the line-of-sight image collection introduce an increased complexity in deploying the cameras inside the building to cover all the possible room locations. Moreover, the expensive hardware for cameras and the issues related to occupant privacy have critically degraded its acceptance and deployment [38,42]. In recent years, Wi-Fi-based proximity sensors are used extensively for calculating the indoor occupancy number $[43,44]$. While using this method, the Wi-Fi network is mandatory in all locations inside a building. In addition, each occupant needs to carry a Wi-Fi enabled device all the time. If the occupants leave their devices in the offices and go to some other places. The Wi-Fi-based sensors will calculate the occupants' positions of their devices but not their actual locations [38]. The errors in calculating the precise occupancy and location detection can arise in this case. Most smart devices include a GPS antenna that is used for locating the positions of their owners. However, the performance of GPS-enabled devices degrades severely inside the buildings because of the signal interferences caused by the building objects [45]. As a result, Bluetooth-enabled beacons came into the application for capturing the movements of users for indoor building environments and have been used widely in recent studies $[3,12,46]$.

\subsection{Need for incorporating the dynamicity of building environments}

After an extensive review of existing applications of occupant behavior modeling [13-37], it is observed that numerous systems have been developed to understand occupant behaviors. Each system is developed after conducting different types of measurements (real site or laboratory) and surveys that focus on incorporating different model variables (e.g. physical, biological and environmental) and human factors for representing different occupant behaviors (e.g. movements, occupancy, body postures, etc.) for different applications of facility management with respect to different types of buildings (residential, commercial, etc.). Consequently, the existing systems for understanding occupant behaviors cannot be compared to one another as each system has unique functionality and scope of inferring the actions using the sensory data is limited to the application. However, Hong et al. [4] provided a DNAS ontology, which acts as a technical framework to standardize the major components (drivers, needs, actions, and systems) required to model occupant behaviors. A DNAS ontology primarily aims to model energy-related occupant behaviors. However, the features which were observed fundamental in DNAS and most of other developed occupant modeling systems are occupants' movements and presence. In fact, the occupants' movements and presence are considered as the prerequisites for any kind of behavior understanding as building occupants can only interact with the building if they are present inside the building [5]. Existing systems in the literature are built by capturing the stochastic and reactive nature of occupant behaviors to model their movements and presence in buildings. Ultimately, these systems contribute to enhancing the understanding of occupant behaviors by increasing the occupancy resolution (i.e. inferring different occupant activities using their movements and presence) for different building monitoring and management applications. However, the existing systems for occupant behavior modeling do not incorporate the information of complex dynamic environments where the building objects (occupants and building locations) evolve over time (see Fig. 2). With the passage of time, the functionalities of the locations in a building often change (i.e. change in semantics or a context) [3]. For example, a room named 'inventory' in a building is now an 'office' having different functionality. Likewise, due to the placement of certain inventory on a specific area of a building, the floor area of a building (a set of rooms and a corridor) became a 'restricted area'. In addition, new walls or infrastructure support may be added in a building $[3,11]$. This will result in a change in the dimensions (i.e. geometry) of building locations (called as spatial changes). The change in the semantics of building locations occurs often in constructed facilities whereas, the spatial changes take place rarely [3]. Such changes need to be incorporated in occupant behavior modeling as a change in the purpose or a position of building locations will result in different behaviors of occupants which ultimately represent different occupant activities [11]. The updated spatial and semantic information about the building locations along with the previous information will contribute to an improved understanding of occupant behaviors with respect to the changes occurred in the building environment [3]. Resulted occupant behaviors after modeling the dynamicity of the building environment can be used for different construction and built environment applications [11] such as; construction resource monitoring for improved safety, managing building spaces based on their utilization, implementing occupant access control system, etc. To address these requirements of dynamic building environments which contain evolving building objects, we have used our ontology graph-based STriDE model.

\section{OBIDE framework}

The proposed integrated framework as shown in Fig. 3 describes occupant behaviors using a DNAS ontology [4] having four major components which are drivers, needs, actions and systems. Drivers represent the factors which stimulate an action to be performed by occupants in a building environment. Needs are the requirements (physical, non-physical) which should be met of occupants in a building to acquire the desired satisfaction. Actions are the interactions or movements of occupants in a building to achieve a certain level of comfort. Systems represent a building and its equipment through which the occupants interact with the building. More information on a DNAS ontology can be found here [4]. After describing the occupant behaviors which need to be modeled, a process of data acquisition takes place to enrich a DNAS ontology with more information required for modeling the occupant behaviors. The additional information is linked with the actions of the occupants which is conventionally collected using different sensors. The type of sensor data and the method using which the data is acquired for modeling the behaviors after enriching a DNAS ontology is completely dependent on the type of application as discussed in Section 2(b). However, for each case-study, occupants' movements and presence are the pre-requisites for any kind of actions which will lead to behavior understanding of occupants as occupants can only interact with the building environment if they are physically present inside the building [5]. If the occupants' movements and presence are modeled correctly, this will increase the occupancy resolution of the model which ultimately helps to infer occupant activities with higher accuracy $[1,3]$. Keeping this minimum level of modeling as a foundation, the STriDE model is used to incorporate the dynamicity of building environment to better understand the occupants' movements (actions) which can later help to infer occupant activities. The STriDE models the building environment as a collection of different building objects (entities). In our case, we have three building entities which are 1. a trajectory (corresponds to occupant location i.e. spatio-temporal point), 2. a location (a physical building location) and 3. an occupant. Each building entity evolves over time under the action of different processes. The life cycle of each building entity is summed up into a series of different states. Each state represents a change in the entity. As shown in Fig. 2, a change can occur in the location, geometry or the semantic (thematic) attributes of a building entity [3,10].

The different changes in building entities as mentioned above can occur independently or simultaneously in a building environment. The 'concepts' are defined in a data model for tagging the building locations with spatio-temporal trajectories of occupants. The data model [11] uses a set of classes and properties from the existing vocabularies for defining the concepts and their relationships which are; 1 . Simple Knowledge Organization System (SKOS), 2. Dublin Core Terms (DCT) 


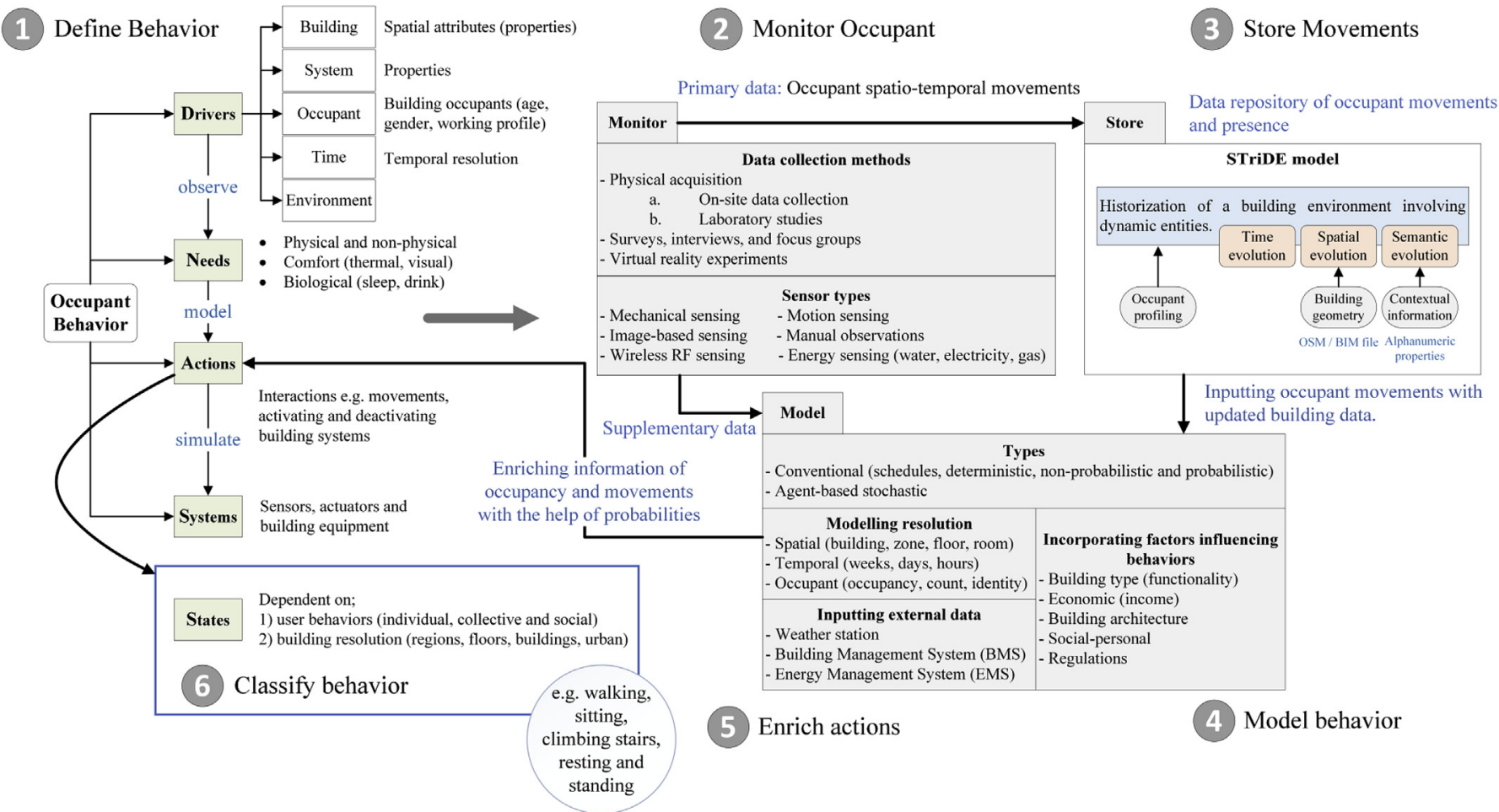

Fig. 3. Generalized OBiDE framework.

and 3. GeoSPARQL (GEO). Using these vocabularies, the concepts are defined and stored in the 'concept scheme'. A concept scheme is a collection of different concepts defined as a hierarchy which correspond to different building locations [12]. The purpose of defining as a hierarchy is to be more precise about the tagging of building locations to spatio-temporal trajectories of occupants [3]. Using a set of different concepts, occupant profiles are created to define the access level of the occupants. For identifying the occupants, the tagged concepts with the occupant trajectory's timeslices (TSs) are compared with the allocated concepts as per their profiles. To model the building environment, the STriDE model keeps track the building entities (see Fig. 2) as well as different relations among them which are;

- the spatial relation; specifies how an entity is in a building in a relation to a reference entity (a room). For example, an entity A (i.e. an object) passes through entity B (i.e. a location) or the geometries of entities A and B overlap each other or touch at a specific point in a building.

- the spatio-temporal relation; specifies how building entities (two rooms, or a room and an occupant) are related to each other at the same time.

- the filiation relation; specifies how building entities are related by ancestry or successor [10]. It defines the succession links which exist between several representations of the same entity at different time instants.

The data model deals with two types of filiation relationships [10]; a continuation (an identity of a building entity remains the same while an entity changes) and a derivation (after a change a new building entity is created from the parent entity). For tracking the evolution of building entities (occupants, trajectories and rooms) and their relations with one another, the model uses the concept of TSs. A TS includes four components which are; an identity, alphanumeric properties (semantic component), a geographical (spatial) and a time component [3,12]. At the occurrence of a change in any component of a TS excluding the identity, a new TS is generated inheriting the components of the last known TS. To show how our model keeps tracking the evolution of dynamic building entities (location, trajectory, and occupant) using TSs, three possible scenarios are described which are;
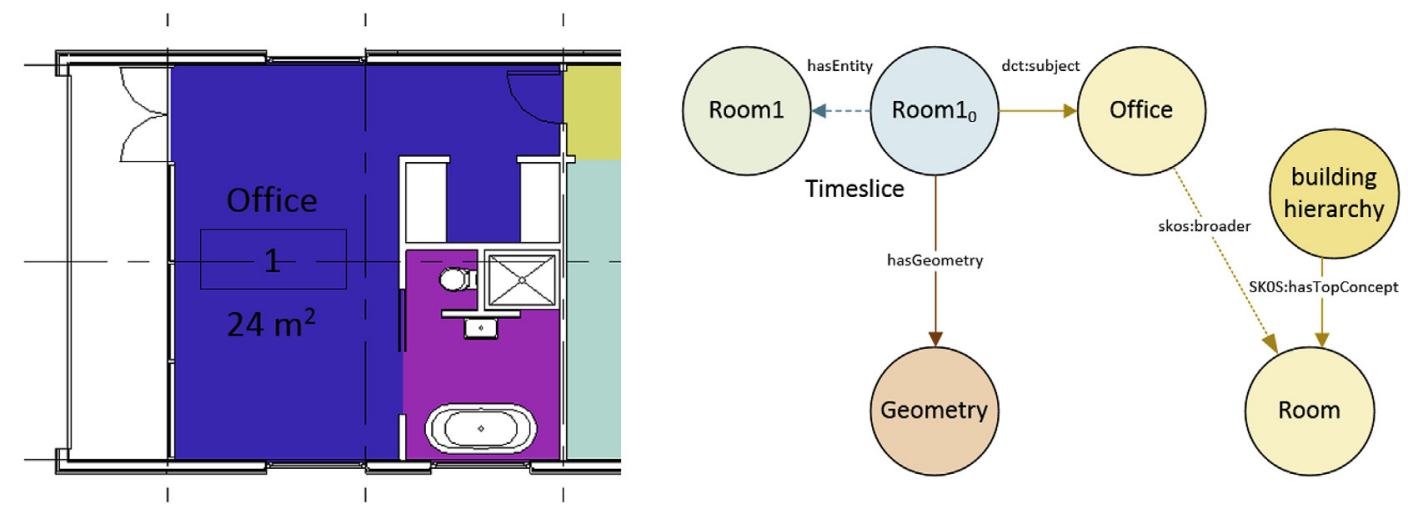

Fig. 4. Building room with its information in a data model. 

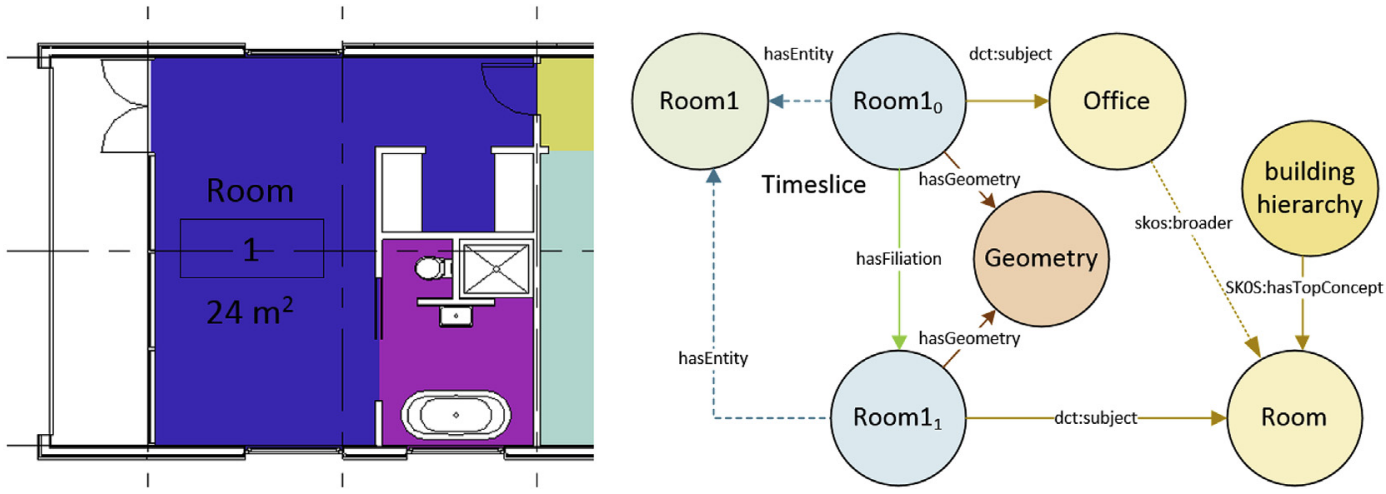

Fig. 5. The functionality of a building room is changed.

1. The functionality of a location is changed (a geographical component); A building room named: Office having a size of $24 \mathrm{~m}^{2}$ as shown in Fig. 4. The room is tagged as an Office in a model having a defined geometry. After a while, a building room named: Office is now a general Room having a same geometry of $24 \mathrm{~m}^{2}$ and tagged as Room with a help of a new TS Room $1_{1}$ in a model having the same geometry as of Office. The transition between an Office to a Room is stored using a filiation link as shown in Fig. 5.

2. The geometry of a building room is changed; A building room named: Room has now a size (geometry) of $28 \mathrm{~m}^{2}$. A new TS is created with a name of Room $1_{1}$ to hold this change in geometry. The change in the geometry between an Office to a Room is stored using a filiation link as shown in Fig. 6.

3. The creation of a new building room; Let's suppose, a room labeled Office is demolished. A new room i.e. Room2 is created as shown in a building model created in Revit Architecture software by Autodesk. A Revit software is used for creating the three-dimensional model of a building [12,47]. A room in Revit is represented using a three-dimensional volume space. A Revit room can be created by going to room tools located on the 'room and area' panel in Revit software (see Fig. 7). Later, this newly created room is labeled by placing the room tag associated with it as shown in Fig. 7. As a building room named: Office is destroyed (see Fig. 8a and b). Initially, its TS Room $1_{0}$ is updated by changing its end date-time stamp to show that a room is no longer exists (see Fig. 8c). Also, two new TSs are constructed which are Room $2_{0}$ and Room $3_{0}$ to show the construction of the two rooms having different geometries (see Fig. 8d). For this case, new rooms are created which are linked to the previous room. Therefore, filiation links are used.

The OBiDE framework aims to add the dynamic context to occupant behaviors' modeling process as context (details about building space, time and environment) should be closely linked with the occupant movements for an enhanced understanding of their actions. After creating a knowledge base of historized movements of occupants using the STriDE model by tracking the evolutions occurring in the building environment, an appropriate modeling technique as per application requirements (agent-based or conventional) can be applied to the movements for calculating the probabilities of different actions of occupants across different building locations. A critical question here is the degree of detail about occupants and the environment which should be included in the modeling stage to attain the targeted understanding of occupant behaviors. These details include occupant profiles, the type and the number of buildings, and the required temporal (e.g. hours, minutes, seconds) and spatial resolutions (e.g. floors, rooms). Our data model can execute an access control system after creating different occupant profiles with the help of concepts as described above. Also, the STriDE model can hold data of multiple buildings with the help of OpenStreetMap (OSM) building files for tagging updated building locations to spatio-temporal movements of occupants. Moreover, for the modeling of occupant trajectories, the temporal resolution is kept maximum i.e. seconds, whereas the spatial resolution is kept to rooms.

Understanding occupant behaviors is a complex phenomenon. It not only involves the process of tracking movements with their dynamic context and computing the probabilities of their different actions inside the building that is one of the pre-requisites of any kind of behavioral analysis but also needs to incorporate several external factors (e.g. weather information, data from building management and monitoring systems, etc.) to study occupant behaviors in more detail by extracting insights about occupant activities from their actions. However, a scope of the proposed framework is kept limited to include dynamicity of the building environment into occupant movements and presence by enriching a DNAS ontology (particularly actions) which can help to infer occupant activities for different facility management applications.

\section{Application of proposed framework}

The proposed framework (see Fig. 3) requires sensory data to include context to occupant movements and presence for performing behavioral analysis. The acquisition of relevant sensor data is based on
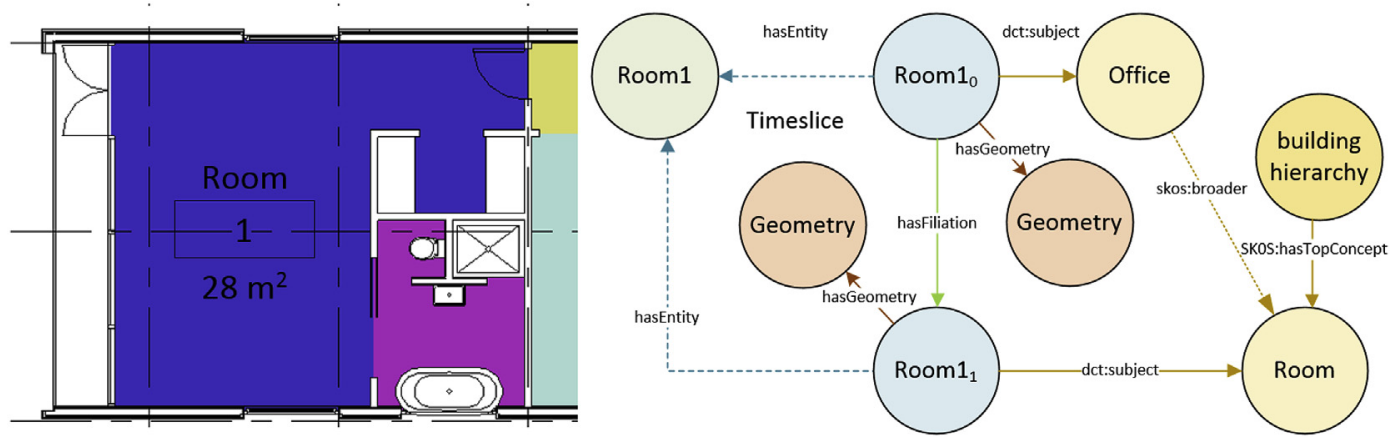

Fig. 6. The geometry of a building room is changed. 


\begin{tabular}{|c|c|c|c|c|c|}
\hline Collaborate & aborate & Manage & Add-Ins & Modify | Place Room & $\bowtie$. \\
\hline \multirow{2}{*}{ I. } & \multirow{3}{*}{$\begin{array}{l}\text { A Model Text } \\
\text { [C. Model Line } \\
\text { [G] Model Group - } \\
\text { Model }\end{array}$} & \multicolumn{2}{|c|}{ 四 Room } & Area & 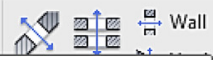 \\
\hline & & \multicolumn{4}{|c|}{ Reom (RM) } \\
\hline in & & & \multicolumn{3}{|c|}{$\begin{array}{l}\text { Creates a room bounded by model elements (such as walls, } \\
\text { floors, and ceilings) and separation lines. }\end{array}$} \\
\hline \multicolumn{3}{|c|}{$\square$ Leader Room: New } & \multicolumn{3}{|c|}{ Press F1 for more help | E Eideo is loading... } \\
\hline
\end{tabular}

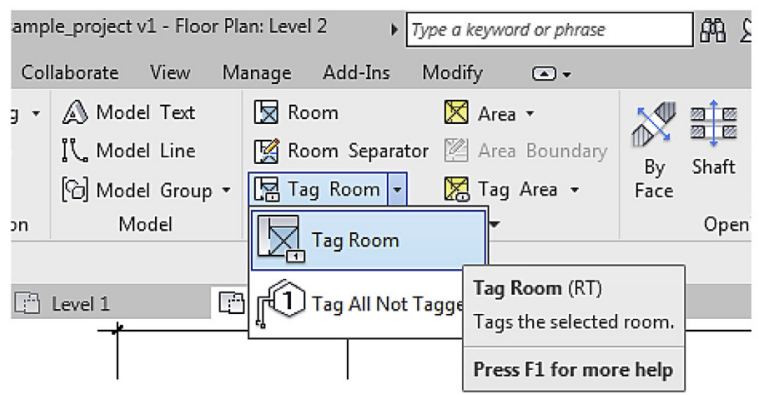
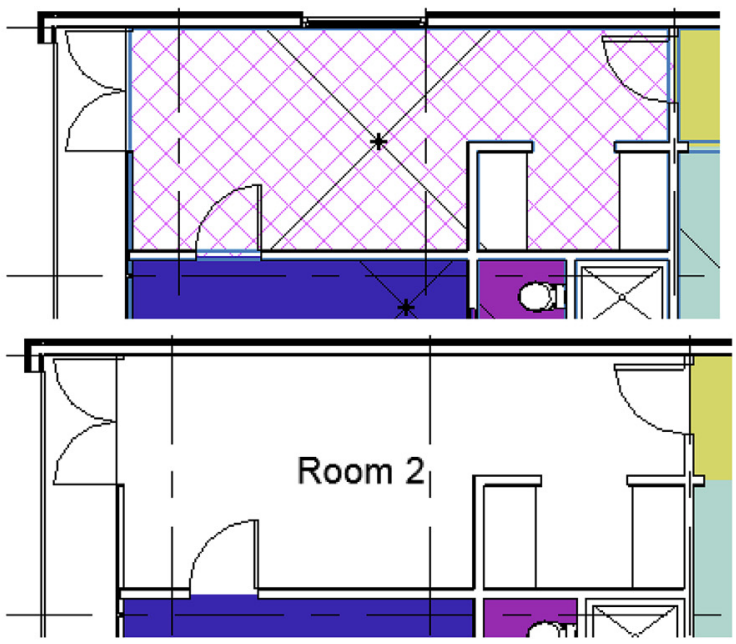

Fig. 7. Creating a room (top row) and tagging a room with a label 'Room 2' in a Revit software (bottom row).

the application requirement. For example, the safety manager of a building requires to monitor the movements of the occupants in a building. In this case, using our OBiDE framework (see Fig. 9), 'driver' is monitoring movements of occupants, 'need' is achieving safety management in a building by identifying unsafe movements, 'action' is tracking movements of occupants using their spatio-temporal trajectories, 'system' corresponds to Bluetooth Low Energy (BLE) beacons for sensor data acquisition deployed in a building and 'states' are 1) static (no movement), 2) normal movement $(0<$ steps $\leq 84$ and $\pi /$ $2 \leq$ angle $<\pi$ ) and 3) risky or unsafe (steps $>84$ angle $\geq \pi$ ). Existing studies [11,12,48-50] suggests that the movement behavior of an occupant can be defined if the values of step lengths and turning angles are calculated for each two successive trajectory data points. The average walking speed of a person range from 1.0 to 1.6 meters per second $(\mathrm{m} / \mathrm{s})$ [49]. Keeping an indoor environment into account, a value of $1.4 \mathrm{~m} / \mathrm{s}$ as a safe walking speed limit that will give us 84 steps
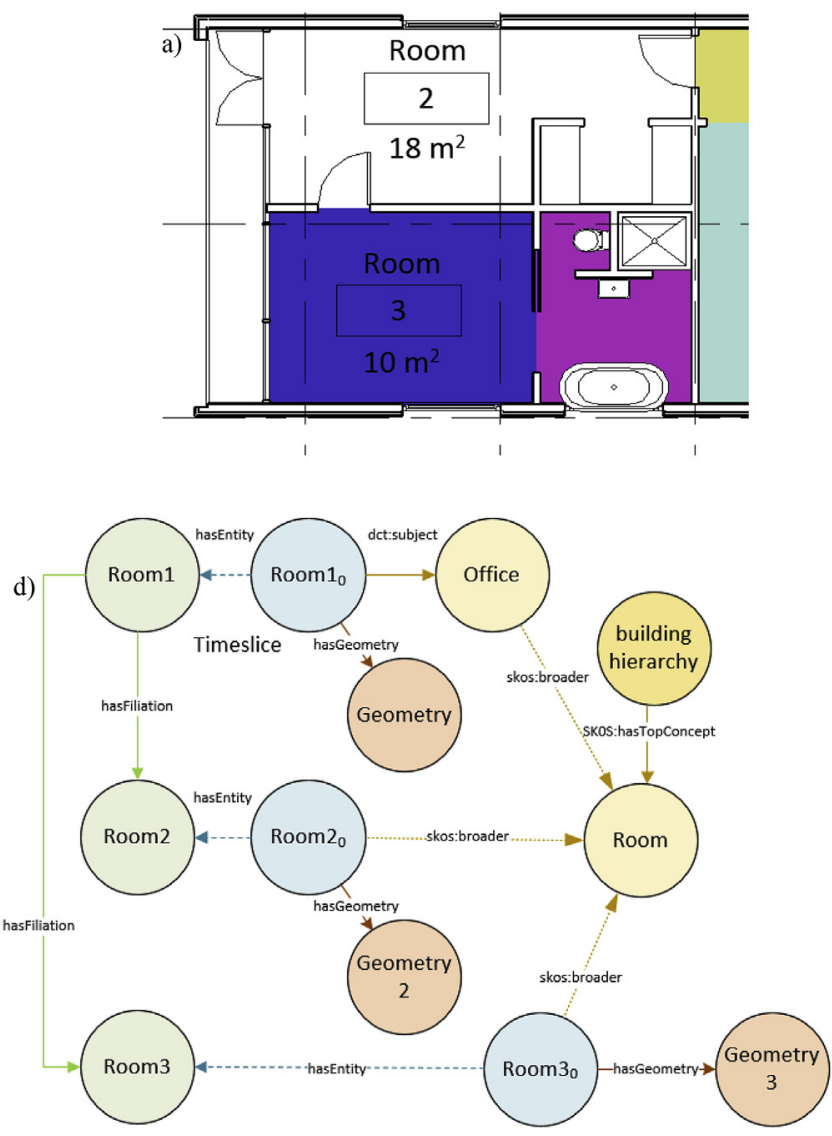

b)

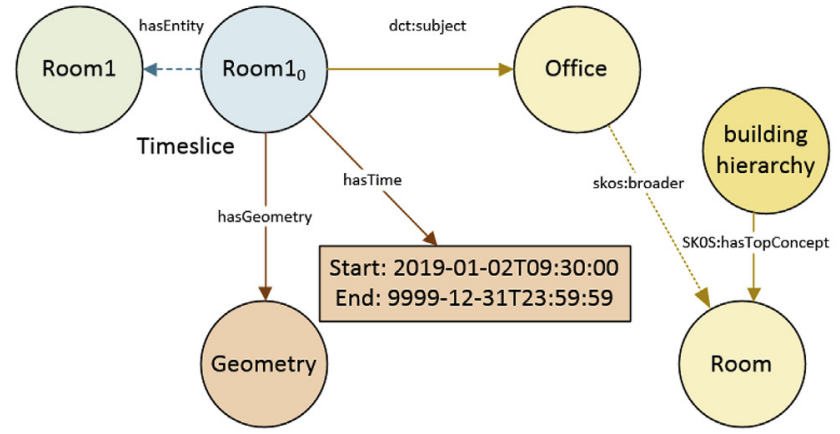

c)

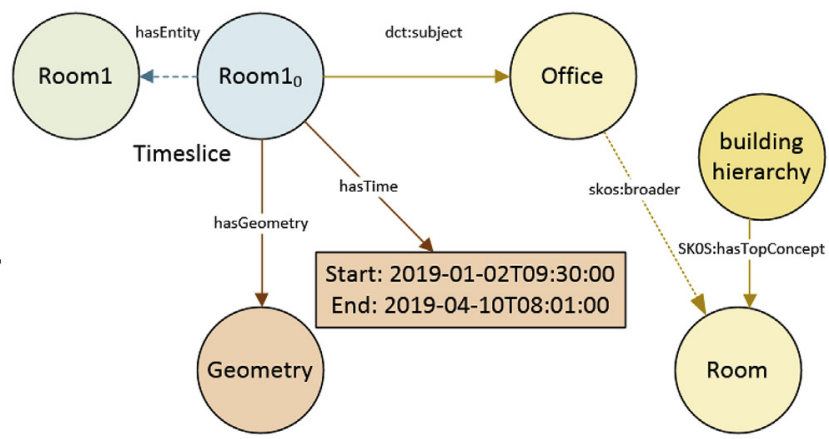

Fig. 8. Storing the information of newly created building rooms in a data model. 


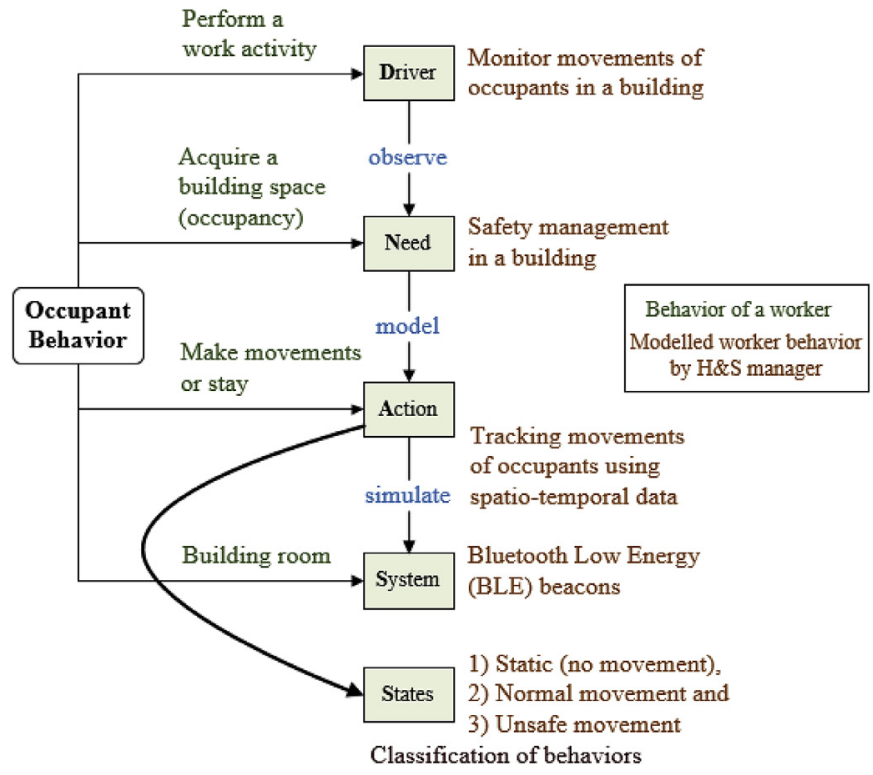

Fig. 9. Application of a proposed framework.

per minute i.e. the sum of step lengths for a minute.

\subsection{Data collection and pre-processing}

For understanding movement-based behaviors for a safety management application, around 200 BLE beacons by Kontakt.io were installed on different building locations (see Fig. 10). The cost of each beacon ranges from 10 to 20 euros [17] and is dependent on the size of the area which needs to be monitored. The deployment plan of the BLE beacons is created using software for covering each building location with a range of at least three beacons by restricting each beacon's signal strength to 5 meters. Approximately 8426 location coordinates (see Table 2 for buildings' information and Table 3 for a dataset description) were collected across different locations using deployed beacons. After sensor data acquisition, the acquired location data is transformed into trajectories after preprocessing (i.e. filtering).

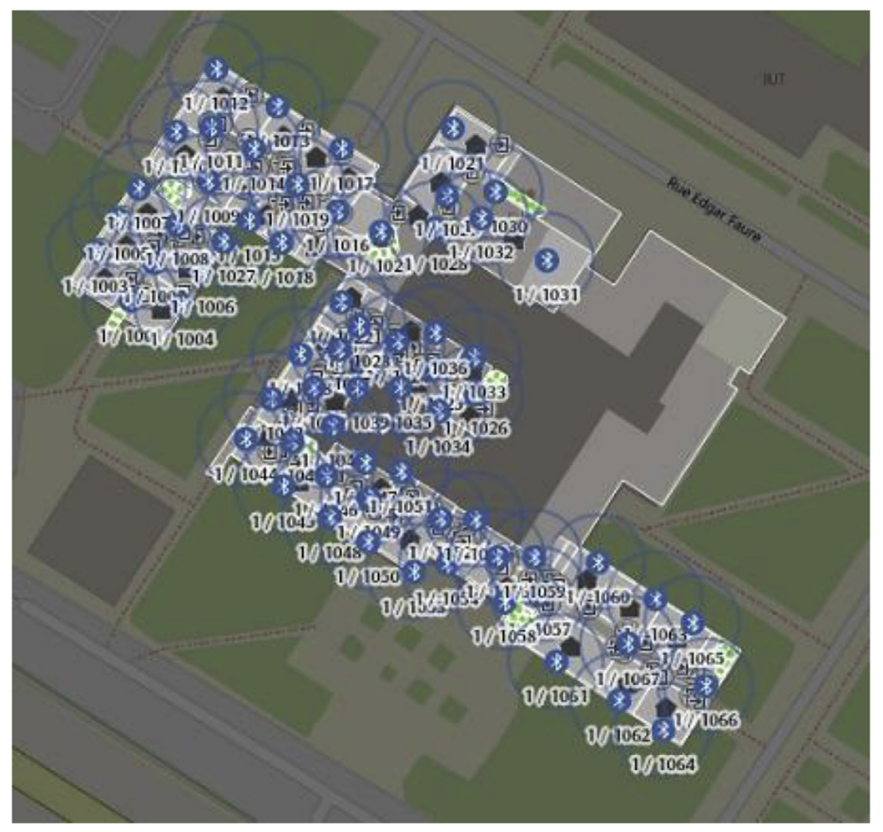

Fig. 10. Deployment of beacons in a building [12,51].
Table 2

Building information.

\begin{tabular}{llll}
\hline No. of buildings & Type of buildings & Occupant type & No. of users \\
\hline 2 & Educational & Students & 11 \\
\hline
\end{tabular}

Table 3

Trajectory dataset information.

\begin{tabular}{cllll}
\hline Dataset & $\begin{array}{l}\text { No. of } \\
\text { trajectories }\end{array}$ & $\begin{array}{l}\text { No. of location } \\
\text { coordinates }\end{array}$ & $\begin{array}{l}\text { Duration of } \\
\text { study }\end{array}$ & $\begin{array}{l}\text { Sampling } \\
\text { frequency }\end{array}$ \\
\hline $\begin{array}{c}\text { Building } \\
\text { users }\end{array}$ & 30 & 8426 & 2 weeks & 5 seconds \\
\hline
\end{tabular}

\subsection{Analyzing the positioning errors}

After preprocessing the spatio-temporal trajectories (see Fig. 11) collected from the BLE beacons, the process of calculating the accuracy of identifying the correct building locations using the building information residing in our data model is executed with the help of queries. The queries were executed for extracting certain user movements which were not correctly acquired by the system.

For instance, at time $t=1$, a user was in Room256. Whereas, at $t=2$ a user is in Room206 (see Fig. 12). Though, these two building locations are not physically connected as observed using building model information. By considering the sampling interval of a trajectory data i.e. 5 seconds, it is not possible to have such fast user movement between two building locations which were not connected. The accuracy of identifying the correct locations by the BLE beacons was around 90\% (see Table 4).

Few locations were not identified correctly because of indoor interferences caused by the building objects and people which resulted in inaccurate recognition of the building locations. Closer investigation [51] to trajectory data revealed that inaccuracies in the system majorly caused because of below reasons;

1. Terminating the mobile application for capturing the BLE beacons signals and relaunching it from other building locations transmitted the geographical coordinates of the previous locations. The is typically resulted because of a delay in the location detection and the transmission of the acquired trajectory geographical coordinates to the server.

2. Few beacons might have fallen from their original positions. These beacons were later mounted to the incorrect places and resulted in erroneous trajectory data points.

Another reason which could cause the incorrect identification of user locations in a dynamic environment is that the geometries of the building locations might have changed over time, but these changes are not fully incorporated in the data model [51]. For instance, a door is constructed between two not-connected locations or a wall is built to split the room into two different locations. However, for our case study that is implemented in the already constructed building, the inaccuracies in the trajectory data resulted because of the former two reasons.

After preprocessing and examining the accuracy of trajectories, the STriDE model is used to add contextual information of a building environment in collected trajectories for mapping actual building locations with each trajectory point (see Fig. 13). As shown in Fig. 13, trajectory timeslices (TrajOfUser1-2, TrajOfUser1-5, etc.) of a User1 are mapped to the corresponding building locations (e.g. Office1, CorridorFloor2, etc.). 


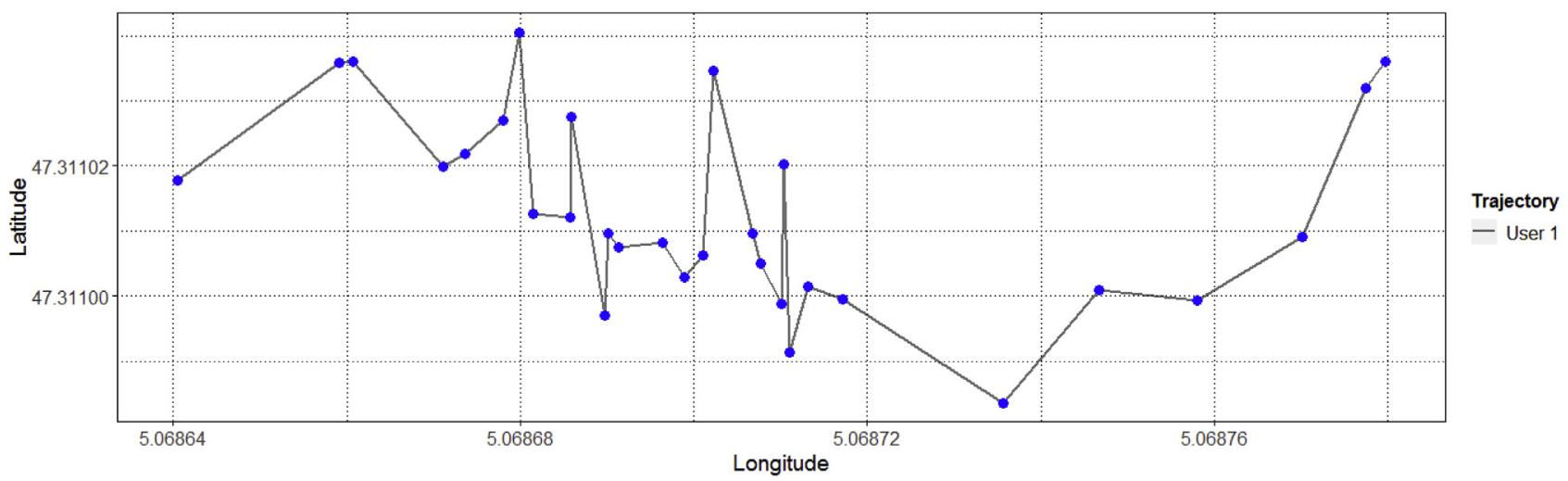

Fig. 11. Spatio-temporal trajectory of a user.

SPARQL Results (returned in $50 \mathrm{~ms}$ )

\begin{tabular}{|c|c|c|}
\hline Trajectory & From & To \\
\hline ¿ dm: User1 & c dm: Room265 & (5) dm: Room206 \\
\hline dm: User1 & 匹 dm: Room206 & dm: Room22 \\
\hline dm: User1 & d dm: Room22 & dm: Room242 \\
\hline d dm: User1 & C dm: Room242 & ds dm: Room22 \\
\hline dm: User1 & d dm: Room22 & (5) dm: Room105 \\
\hline
\end{tabular}

Fig. 12. User movements between not-connected building locations.

Table 4

System accuracy of identifying correct locations.

\begin{tabular}{lll}
\hline $\begin{array}{l}\text { Detected user locations in } \\
\text { buildings }\end{array}$ & $\begin{array}{l}\text { Correctly identified building } \\
\text { locations }\end{array}$ & System Accuracy \\
\hline 150 & 136 & $90 \%$ \\
\hline
\end{tabular}

Table 5

Confusion matrix for a 3-state HMM evaluation.

\begin{tabular}{lllll}
\hline \multirow{2}{*}{ No. of test samples = 2132 } & \multicolumn{3}{l}{ Predicted States } \\
\cline { 3 - 5 } & & $\boldsymbol{S}_{1}$ & $\boldsymbol{S}_{2}$ & \multicolumn{1}{l}{$\boldsymbol{S}_{3}$} \\
\hline \multirow{2}{*}{ True States } & $\boldsymbol{S}_{1}$ & 1066 & 23 & 0 \\
& $\boldsymbol{S}_{2}$ & 30 & 810 & 75 \\
& $\boldsymbol{S}_{3}$ & 6 & 17 & 105
\end{tabular}

$\mathbf{S}_{1}=$ Stay, $\mathbf{S}_{2}=$ Normal movement, and $\mathbf{S}_{3}=$ Risky movement

SPARQL Results (returned in $55 \mathrm{~ms}$ )

\begin{tabular}{|c|c|c|}
\hline Trajectory & UserName & Location \\
\hline$G \mathrm{dm}$ : TrajOfUser1-1 & dm: User1 & OutdoorPathway \\
\hline (U) dm: TrajOfUser1-2 & dm: User1 & Office1 \\
\hline (5) dm: TrajOfUser1-3 & dm: User1 & StorageRoomFloor2 \\
\hline d dm: TrajOfUser1-4 & dm: User1 & MeetingRoomFloor2 \\
\hline dm: TrajOfUser1-5 & dm: User1 & CorridorFloor2 \\
\hline
\end{tabular}

Fig. 13. Occupant movements transformed into a contextually-enriched trajectory.

\subsection{HMM-based trajectory analysis}

For modeling the behaviors to recognize and categorize the movements into different states, many types of non-probabilistic and probabilistic approaches exist in the literature such as Bayesian dynamic models and clustering techniques, state-based models such as simple Markov chains and HMMs, patterns matching algorithms and deep learning-based techniques [12]. Though, HMMs-based method is selected for our application as statistical HMMs describe occupant movements as a series of Markovian stochastic processes where the probability distribution of a future state (i.e. safe or unsafe behavior or a next location) of a stochastic (i.e. a random) process (a trajectory in our case) is only dependent on its current state or a current location which disregards the requirements of including the preceding states and ultimately minimal training data is required [52].

An HMM has three fundamental properties [52] which define it. 1) It assumes that the observation

$O=\left\{o_{1}, o_{2}, o_{3}, \ldots, o_{T}\right\}$ was produced by a process at time $t$ whose state $S_{t}$ is hidden from the user. 2) The hidden state process fulfills the Markov property where the current process state $S_{t}$ is exclusively dependent on only the previous state $S_{t-1}$. 3) The hidden state variable can only take $T$ integer values $\{1,2,3, \ldots, T\}$. Generally, an HMM ' $\lambda$ ' is described using three main parameters which are written as 3tuple $\lambda=(A, B, \pi)$. Where, $A=\left[a_{11}, a_{12}, a_{13}, \ldots, a_{n n}\right]$ is the transition probability matrix. Each $a_{i j}$ in $\left[a_{11}, a_{12}, a_{13}, \ldots, a_{n n}\right]$ represents the probability of moving from state $i$ to state $j$. The sum of transition probabilities should be equal to $1 . B=b_{i}\left(o_{t}\right)$ is the emission probability matrix is also known as observation likelihood, where each $b_{i}\left(o_{t}\right)$ represents the probability of an observation $o_{t}$ being generated by a state $i$ and $\pi=\left\{\pi_{1}, \pi_{2}, \pi_{3}, \ldots, \pi_{N}\right\}$ is the vector of the initial state probabilities. For training the HMMs, Baum-welch algorithm [52] is used which adjusts the model parameters $(A, B, \pi)$ as discussed above to maximize the probability of the observation sequence given the model i.e. $P(O \mid \lambda)$. For inputting the initial probabilities for the hidden states which are; 1 . static (no movement), 2. normal movement $(0<$ steps $\leq 84$ and $\pi /$ $2 \leq$ angle $<\pi$ ) and 3) risky movement (steps $>84$ angle $\geq \pi$ ) are given equal probability of occurrence i.e. $\pi=\left\{\pi_{1}, \pi_{2}, \pi_{3}\right\}=\left\{\frac{1}{3}, \frac{1}{3}, \frac{1}{3}\right\}$. For inputting the movement states into HMMs, Gamma distribution [53] for step lengths and Von Mises distribution [54] (also known as the circular normal distribution) for turning angles are used. Out of 30 trajectories, 25 trajectories of randomly selected users were used for training the HMM. Whereas, 5 trajectories were used for the validation purpose.

\subsubsection{Model checking}

After training the HMM using 25 trajectories of users, the trained model is evaluated against the training dataset. To evaluate the general goodness of the 3-state trained HMM, pseudo-residuals [55] (also known as quantile residuals) are calculated using a trajectory 

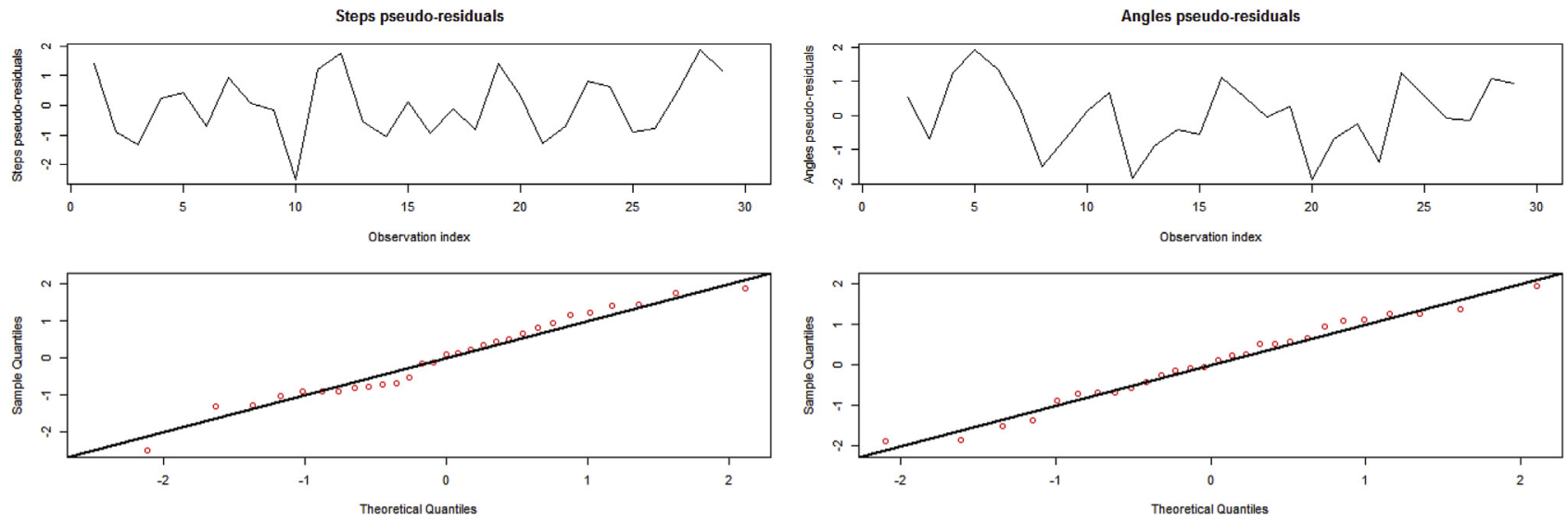

Fig. 14. Time series (top) and qq-plots (bottom) of the pseudo-residuals of the 3-state model for step lengths (left) and turning angles (right).

sequence $O=\left\{o_{1}, o_{2}, o_{3}, \ldots, o_{T}\right\}$ for verifying whether the trained HMM is a true data generating process of a user trajectory or not. The residuals are what is left over after fitting a trained model and their values are equal to the difference between the trajectory observations and the corresponding fitted values [55-57]. If the trained HMM model fits the data well, the data points in the qq-plot will be closer to the straight line and deviations of the points from the normality will indicate a lack of fit [55]. The pseudo-residuals of the 3-state HMM fitted to the trajectory data of an occupant with the theoretical quantiles on the horizontal axis are displayed in Fig. 14. As shown in Fig. 14, the trained HMM is nicely fitted for the observation trajectory dataset and has few deviations from the straight line (i.e. the normality). However, the degree of goodness of a model to incorporate all the possible variations in the trajectory data can be improved by increasing or decreasing the number of hidden states. For more details on pseudo-residuals, see Zucchini et al. [55] research.

\subsubsection{State decoding for model validation}

Once the HMM is fitted using the training trajectory dataset, different test sequences from the trajectories of users are classified into three states which are $S_{1}, S_{2}$ and $S_{3}$ using the Viterbi algorithm [52]. The Viterbi algorithm performs a global decoding process and generates the most probable sequence of states which have produced the observations under the trained HMM model [52]. As an example, Fig. 15 shows the classification of one of the test sequence $O=\left\{o_{1}, o_{2}, o_{3}, \ldots, o_{T}\right\}$. The resulted states' sequences (one of them is shown in Fig. 15) is compared to the ground truth data which is collected by manually extracting the stays, normal and risky movements of the users from their trajectories using the values of step length and turning angle. Step length is computed using the Haversine distance formula which is one of the commonly used methods $[49,50]$ of calculating the geographic distance between the two geo-locations $\left(x_{t}, y_{t}\right)$ and $\left(x_{t+1}, y_{t+1}\right)$ as below;

$d=2 r \sin ^{-1}\left(\sqrt{\sin ^{2} \frac{x_{t+1}-x_{t}}{2}+\cos x_{t} \cos x_{t+1} \sin ^{2} \frac{y_{t+1}-y_{t}}{2}}\right)$

In the above formula, ' $r$ ' is the radius of the earth. Whereas, turning angle in radians is computed as the change in bearing $b_{t}$ as $b_{t}=\operatorname{atan} 2\left(y_{t+1}-y_{t}, x_{t+1}-x_{t}\right)$ between the time intervals $[t-1, t]$ and $[t, t+1]$. Using these parameters, ground truth data is manually constructed. Later, a confusion matrix [58] is computed Table 5 by inputting the predicated states of the trained HMM and the ground truth states' data for describing the performance of the trained HMM classification model. Finally, a precision-recall analysis [58] is conducted after computing the confusion matrix for determining the reliability of the trained model. The precision is defined as the ratio of how much of the predicted data is correct. Whereas, recall is the ratio of how many of the actual trajectory states were predicted [58].

Using the R library of Caret package [59], the actual and predicted values were inputted, and the precision-recall parameters were extracted. The overall precision of the model was $83 \%$, whereas the recall of the model was $89 \%$.

\subsection{Visualizing movement states using BIM}

For the sake of the proof-of-concept demonstration of HMM-based analysis, a single trajectory is decoded into three different states $\left(\mathrm{S}_{1}, \mathrm{~S}_{2}\right.$ and $\left.\mathrm{S}_{3}\right)$ which corresponds to the movements of a user in a building in Fig. 15. The decoded trajectory needs to be linked with the building location from where it was captured [3]. For visualizing the latest classified movement states resulted from an HMM by analyzing a user trajectory, a Building Information Modeling (BIM)-based software i.e. Autodesk Revit Architecture is used. The BIM approach is selected because existing literature recognizes it as a 'future IT solution' and favored over traditional three-dimensional Computer-Aided Design (CAD) approaches [12] as it is an efficient way of information management during the building lifecycle [3]. One of the major benefits of using Revit software is that it is an open-sourced solution [12] and offers the user-customized functionalities using its external plug-ins through its Application Programming Interface (API). For visualizing the decoded states of a trajectory, a Revit plug-in named 'Dynamo' is used. Dynamo is a visual programming tool which gives the ability to define pieces of logic using visual scripting by minimizing the requirement of extensive programming [60]. Dynamo enables us to construct visual programs (known as graphs) by connecting 'nodes' with 'wires' (also called as connectors) for specifying the logical flow of information [60]. Our Dynamo graph consists of three main steps as shown in Fig. 16. At first, a list of all the locations of a building model which were tagged as 'rooms' in a Revit software is extracted into Dynamo. This list is compared with the information of building locations having risky movements obtained from the excel sheet that is resulted by our prototype system. The risky movements are quantified based on their percentage of occurrences in a trajectory. For instance, in

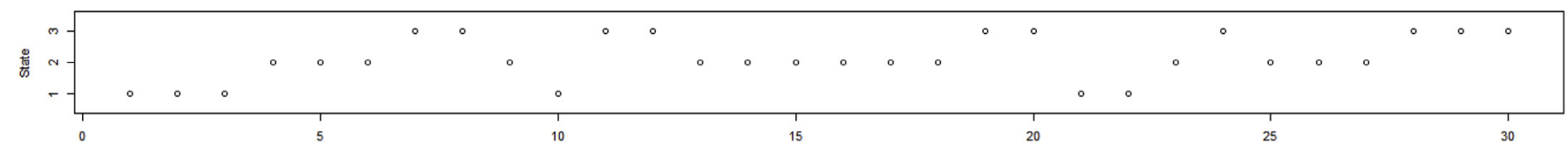

Fig. 15. Decoded sequence of states $\left(S_{1}, S_{2}\right.$ and $\left.S_{3}\right)$ of the trajectory observations of a user. 


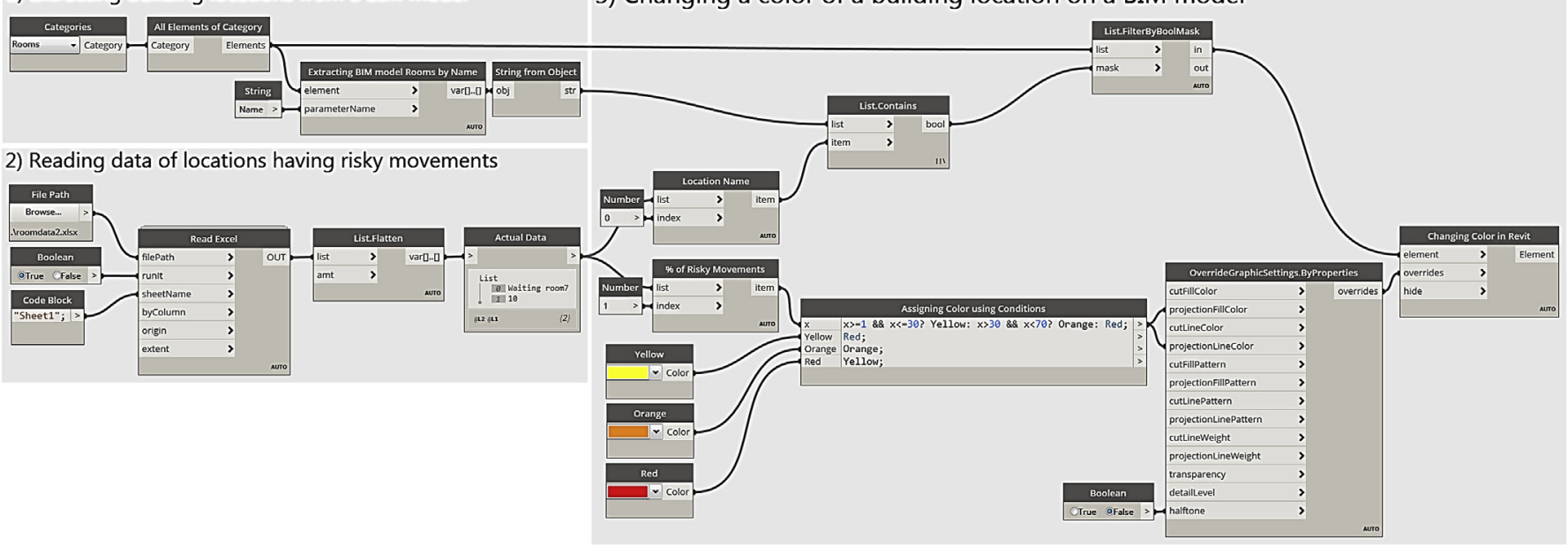

Fig. 16. A Dynamo graph for visualizing different colors against risky movements on a BIM model. (For interpretation of the references to color in this figure legend, the reader is referred to the Web version of this article.)

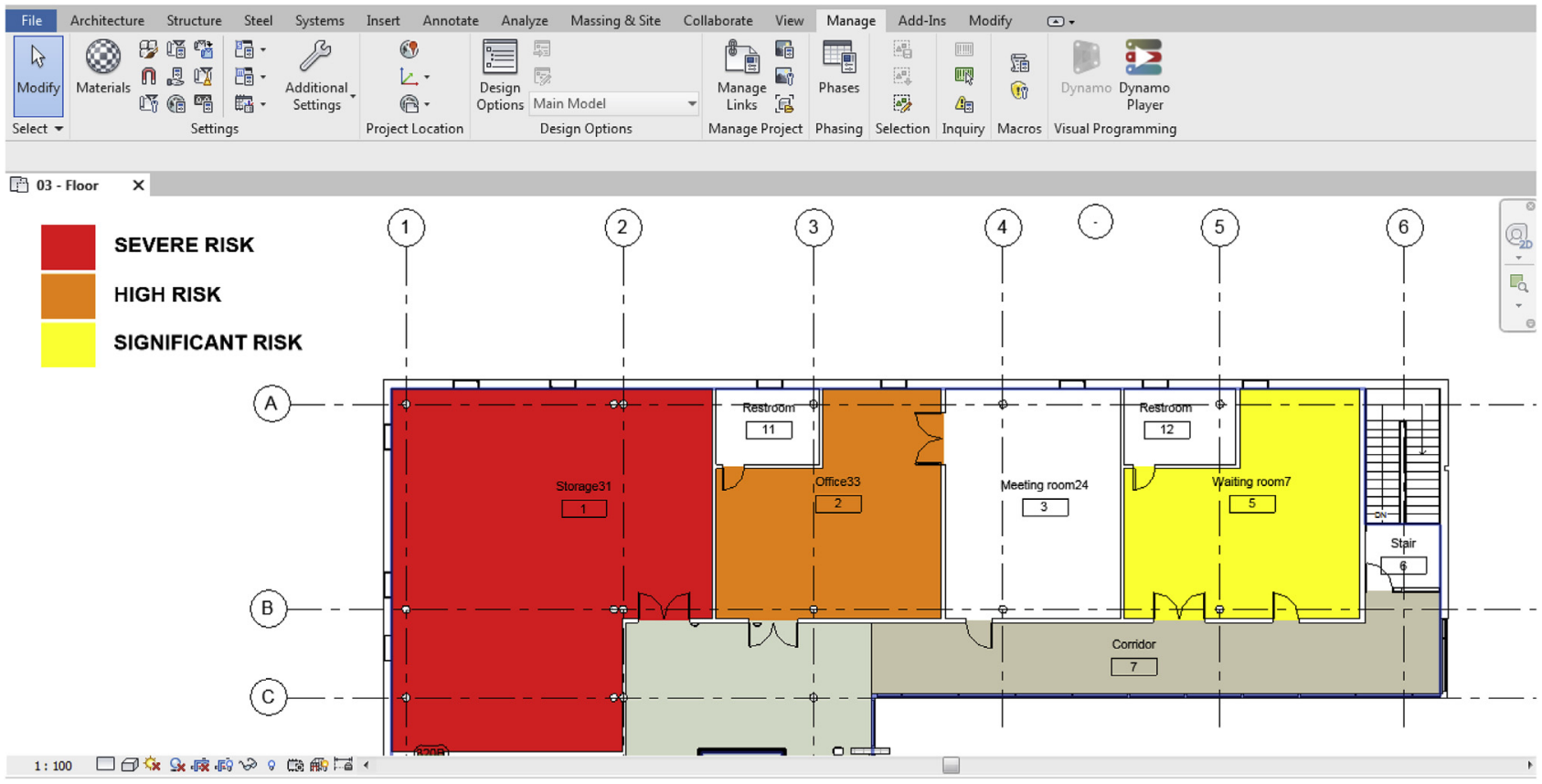

Fig. 17. Visualizing different types of risky movements on a building model in Revit software. Red for $70 \%$ or more, Orange for greater than $30 \%$ and less than $70 \%$, and a Yellow for less than or equal to $30 \%$ occurrences of risky locations in a trajectory. (For interpretation of the references to color in this figure legend, the reader is referred to the Web version of this article.)

Fig. 15, a trajectory having 30 sample points is shown which spans over $2.5 \mathrm{~min}$. An HMM has categorized this trajectory into 3 movement states. Out of 30, 10 states were identified as 'risky states'. The percentage frequency of risky states is computed by dividing the number of occurrences of risky states by the total number of trajectory points and multiplying it by 100 i.e. $\left(\frac{10}{30} \times 100\right)$, a value of 33 is achieved for visualization. To quantify the calculated percentage, three different colors are used for generating the visualization which are; Red, Orange and, Yellow (see Fig. 17). The color scheme is designed using the OSHA color code standard for safety management at work [61]. The red color is used for visualizing such locations which have the highest percentage of risky movements i.e. 70 or more whereas, percentages less than and equal to 30 are shown in yellow color, and percentages greater than 30 and less than 70 are shown in orange color. The range of values for defining different colors was constructed for the sake of demonstrating the functionality of a prototype system to show the criticality of locations by changing their colors on a BIM model. These values can be altered accordingly based on user preferences. To show a process of decoding and visualization using a BIM model, a single user trajectory of short time duration is used. However, in case of multiple and long trajectories of several occupants which are collected throughout a day, the process will remain the same as described above but will be repeated for each individual trajectory. In the end, to assign the color to a location, a function of the averaging needs to be performed on the calculated percentages to acquire a unique value for representing different trajectories on a BIM model. In the 3rd step of our Dynamo graph, conditions for the color assignment are written for visualizing different types of risky locations on a BIM model. The descriptions and the functionalities of the nodes which were used for constructing our Dynamo graph (see Fig. 16) can be found in [60].

An example to understand the impact of change in the contextual information of a building on user behaviors; As discussed in Section 3, for 

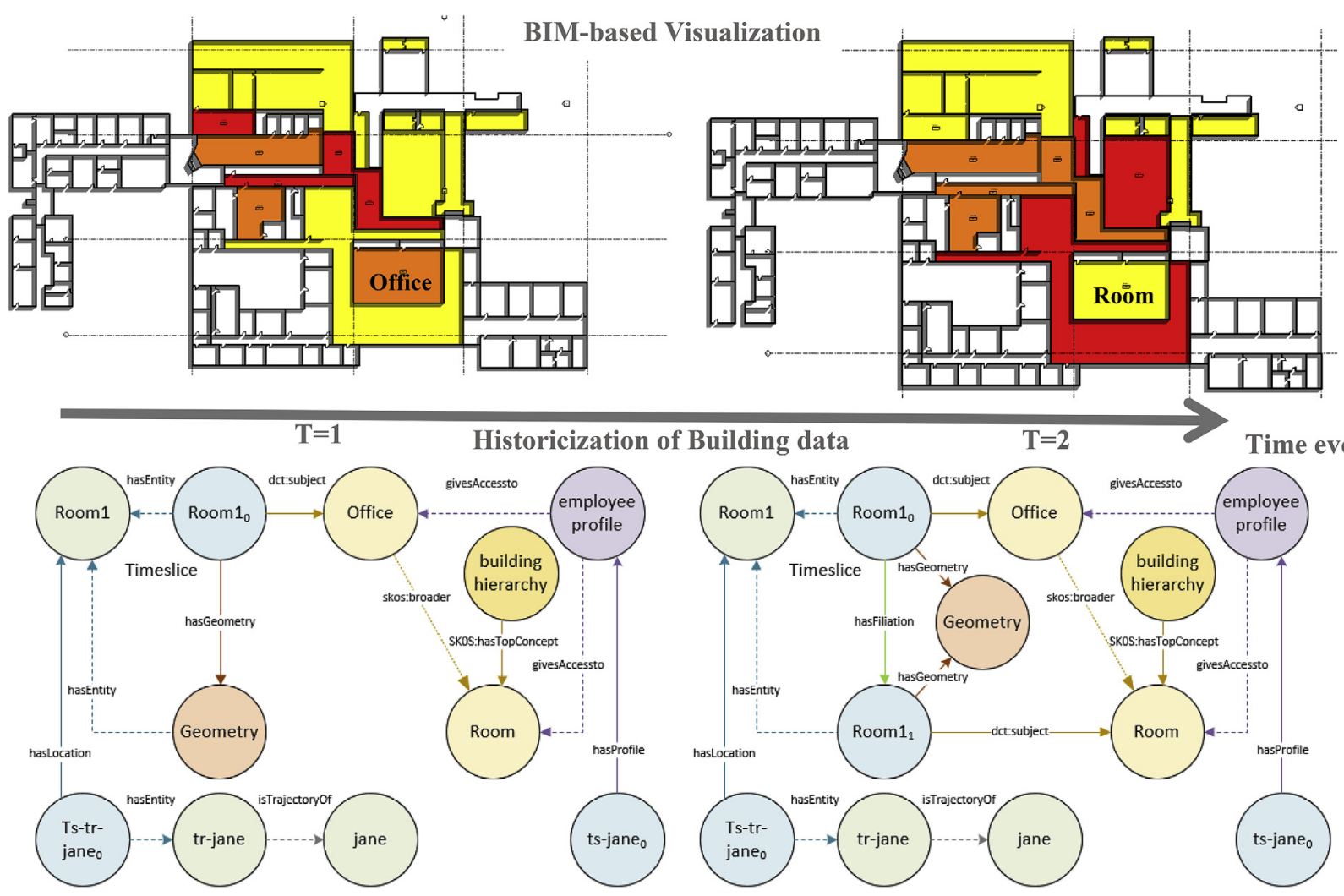

Fig. 18. Change in the functionality of a location (an Office is now a general Room) at $t=2$ resulting in different movements in a building (top row). Maintaining the historicization of building evolution in a data model (timeslices are in Blue, entities are in Green, SKOS concepts are in Yellow, a profile is in Purple and the geometry is in Red (bottom row). (For interpretation of the references to color in this figure legend, the reader is referred to the Web version of this article.)

tracking the evolution of building objects (occupants, trajectories and locations), the STriDE model uses the concept of timeslices (TSs). To show the an application of the proposed framework to hold the building evolution with the contextually-enriched trajectories to study movement behaviors for safety management, let's suppose we have a building from where the spatio-temporal data is collected (as discussed above) and the purpose of one of the building locations is changed (see Fig. 18). The location 'office' is now a 'general room'. As the STriDE model uses 'concepts' for describing the building locations. In Fig. 18, there is a hierarchy of SKOS concepts. It has a skos:has TopConcept connects skos:Concept room. Two skos:Concept (office and room) are defined. All these concepts form a hierarchy. Also, there is a profile named employee Profile which gives access to all the concepts (building locations). As soon as the functionality of a room (i.e. a context) is changed, a new TS is created. For instance, a user Jane is an entity of a TS ts-jane $e_{0}$ and her position is tracked by a trajectory tr-jane. We can observe by a link between tr-jane ${ }_{0}$ and room 1 that Jane is in room1. The entity room 1 was initially an office as suggested by the dct: subject link of tr-room $1_{0}$ towards the concept office. Later, this room is changed as a room having the same geometry as of the office represented as tsroom $1_{1}$.

\section{Discussion}

The objective of this study is to facilitate the development of new systems to enable the standardization of occupant behaviors' descriptions by incorporating the real-life dynamicity of building environments during simulations for an improved understanding of occupant behaviors [11]. To address this objective, a framework named 'OBiDE' is proposed (see Figs. 3 and 19) which offers; 1 . mapping the dynamicity of evolving spatial and contextual building environment's information with occupants' movements, 2 . provides a centralized knowledge base to hold the occupant spatio-temporal movements tagged with relevant contextual information for understanding mobility-based behavioral interactions of occupants, 3. data enrichment of DNAS ontology [4] to enrich occupant behaviors' information using the results of a state-ofthe-art machine learning model in the form of 'hidden states' for calculating the probabilities of the occupant actions and later feeding this information into a DNAS ontology (component: actions) for detailed understanding of occupant behaviors by finding the correlations and patterns in them.

The proposed framework is implemented using ontology-based data model which offers flexibility for further expansions and interoperability for the enrichment of occupant behaviors' descriptions with supplementary data sources to complement the process of occupant behavioral analysis. The application of the proposed framework is described using a case-study for safety management in a building by monitoring the movements of occupants across different locations. For capturing the user movements, in the literature, there exist different types of sensors which provide proximity-based services and coarsegrained location detection for indoor and outdoor building situations as discussed in Section 2(c). For this study, BLE beacons are used for location data acquisition of the building users. The selection of this location acquisition technology is purely done based on its usability explored in the literature [46,51], less cost of deployment and availability for the implementation. It should be noted that proximity (i.e. being close to a certain building object) is linked to the building location where exactly a person is but it is not necessarily the same [51]. An accurate building location of an occupant corresponds to an absolute value of the geographical coordinate system (e.g. longitude and latitude pair values) is more than just proximity [51]. For instance, the Bluetooth signals of beacons fluctuate significantly because of the physical obstacles, the material of walls and ceiling which cause the multipath effects $[46,51]$. These external interferences introduce fluctuations in 


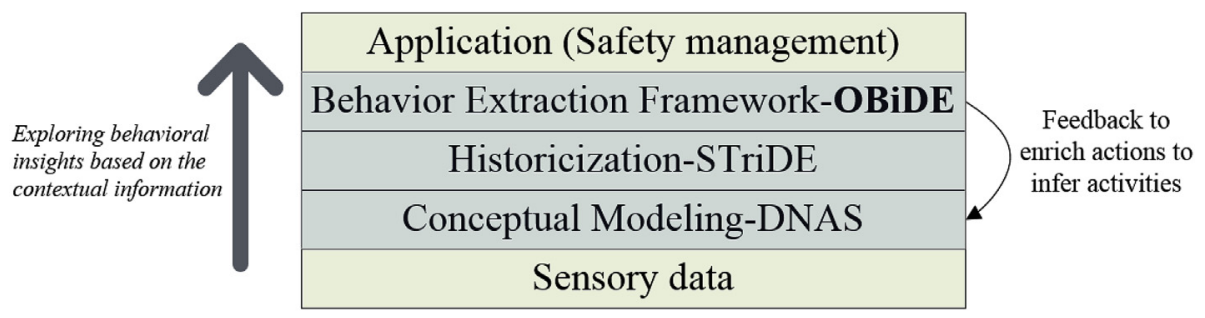

Fig. 19. Different stages in OBiDE framework.

the signal strength values of beacons resulted by the propagation diffraction, reflection, and scattering which ultimately complicates the process of determining the precise location information of occupants in real environments [46]. The process of collecting the precise proximity data of occupants using beacons gets more complicated as the resolution of system increases. The overall system resolution (see Fig. 1) is based on spatial (e.g. building, floor, room), temporal (e.g. hours, minutes, seconds) and occupancy resolution (i.e. occupancy detection, occupancy count, the identity of occupants and inferring the activities of occupants) [62]. As the resolution of the developed sensors increases, the building areas get smaller, building users get more recognizable individually and eventually, their activities can be inferred [51]. For developing an application using the proposed framework, the data model holds the information at room level that is collected per second using beacons for identifying each user across different building locations using their unique identifications. However, the accuracy of precise location detection will be degraded if the spatial resolution increases from room level to areas within the rooms [46,51].

The spatio-temporal data collected using BLE beacons is then preprocessed, transformed into trajectories and contextually enriched by mapping the updated location information with each trajectory point. The contextually-enriched trajectories are stored to build a knowledge base to study occupant behaviors. Later, a probabilistic model i.e. Hidden Markov Model (HMM) is applied on stored trajectories for computing the probabilities of the occurrences of different types of movements. For the categorization of movements, information of the step length and the turning angle is used as these two parameters categorize the mobility [46]. The movements of occupants are classified into three states which are static (stay location), normal and risky by defining the values of step lengths and turning angles for hidden states. Lastly, the most probable latest states of building locations are extracted by analyzing the movements of occupants and shown in Fig. 17 using different colors on a building model.

An HMM has decoded a user trajectory into three states which are; stay locations, normal movements, and risky movements. However, for visualization, only risky states were further quantified and visualized as identifying unsafe movements having run segments and many turnings is the priority for safety managers to take necessary actions in real-time [12]. These unsafe movements can potentially result in an accident if not controlled [12]. Whereas, the other two decoded states of an HMM depict normal movement behaviors of users in a building. A BIM-based software i.e. Revit Architecture is to display movements of occupants. The motivation behind using a BIM-based approach is that it is the universal Architecture, Engineering and Construction (AEC) industry standard and widely used and preferred for maintaining the information a building throughout its lifecycle [12].

For already constructed buildings, the geometry of the locations does not change frequently. Whereas, the purpose of locations in a building keeps changes with a passage of time as per the building requirements. The application of our data model for keeping track the building evolution [63] is demonstrated in a way that the functionality of a room in a building is changed. This change in the contextual information of a location resulted in different movements inside a building. For example, Fig. 18 at $T=1$ shows a tagging of a spatio- temporal trajectory of a user 'Jane' before the location undergoes evolution in the functionality. Whereas, at $T=2$, a building location 'an office' is changed into a general 'room'. This process of evolution is stored in a data model by generating a new TS (i.e. Room $1_{1}$ ). The generated TS have a filiation relation with an office TS (i.e. Room $1_{0}$ ). However, the same geometry as of office is tagged with a new TS (i.e. Room $1_{1}$ ). In this way, the evolution in the contextual information is stored inside in our data model.

\section{Conclusion}

Occupant behavior is a crucial and often overlooked factor in attaining building performance goals such as reducing the consumption of building resources (e.g. energy, materials, etc.). Though advanced smart building technologies integrate different building subsystems to optimize the usage of the building resources. But occupant behaviors (e.g. actions) if not incorporated in modeling can significantly impact the overall performance of a building. The buildings are subject to constant change, where the locations evolve over time in terms of geometry and contextual information during a building lifecycle. This makes the data collection of occupants as well as the building infrastructural changes a continuous process for the exploration of occupant behaviors using a building context. This study has presented an integrated OBiDE framework to maintain the dynamicity of a building environment in a data model and fusing the occupants' movements and presence data (the pre-requisites for any type of occupant behaviors' understanding) with the most updated building information.

Managing the safety of occupants in a building by 1 . capturing their spatio-temporal data, 2. transforming it into contextually-enriched trajectories, and 3 . classifying the trajectories into three different movement states which enriches a DNAS ontology for understanding their mobility-related behaviors is one of the possible use-cases of the OBiDE framework. Moreover, the risky movement states of the occupants were later quantified based on their percentage of occurrences in a trajectory and visualized using a BIM software to provide safety managers a fusion of information extracted from the trajectory data mapped with the updated building architectural model to deliver insights about movements of occupants using a building context. Consequently, building locations with risky movements can be easily identified, and necessary actions can be taken accordingly by the safety managers.

The OBiDE framework which tracks spatio-temporal data of occupant generated in a dynamic environment establishes a basic reference model that can not only be used for presented safety management application but also for space utilization analysis and energy performance simulations where the decision-making processes are performed based on the occupant movements across different locations in buildings. However, additional behavioral occupant datasets should be collected that could allow an improved understanding of a dynamic building environment for enhancing the building overall performance by increasing the occupants' productivity, environmental quality in a building, and functionality of building spaces. 


\section{Acknowledgements}

The authors thank the Conseil Régional de Bourgogne-FrancheComté and the French government for their funding.

The authors also want to especially thank the director of the l'IUT de

Dijon-Auxerre for allowing us to host the living lab

and Orval Touitou for his technical assistance to this research work.

\section{References}

[1] A. Wagner, W. O'Brien, B. Dong, Exploring Occupant Behavior in Buildings, Methods and Challenges, Springer, 978-3-319-61464-9, 2018https://doi.org/10. 1007/978-3-319-61464-9.

[2] S. Chen, W. Yang, H. Yoshino, M.D. Levine, K. Newhouse, A. Hinge, Definition of occupant behavior in residential buildings and its application to behavior analysis in case studies, Energy Build. 104 (1) (2015) 1-13 https://doi.org/10.1016/j. enbuild.2015.06.075.

[3] M. Arslan, C. Cruz, D. Ginhac, Semantic Enrichment of Spatio-Temporal Trajectories for Worker Safety on Construction Sites, Personal and Ubiquitous Computing, 2018, pp. 1-16 https://doi.org/10.1007/s00779-018-01199-5.

[4] T. Hong, S. D'Oca, W.J. Turner, S.C. Taylor-Lange, An ontology to represent energyrelated occupant behavior in buildings. Part I: introduction to the DNAs framework, Build. Environ. 92 (2015) 764-777 https://doi.org/10.1016/j.buildenv.2015.02. 019.

[5] D. Yan, W. O'Brien, T. Hong, X. Feng, H.B. Gunay, F. Tahmasebi, A. Mahdavi, Occupant behavior modeling for building performance simulation: current state and future challenges, Energy Build. 107 (2015) 264-278 https://doi.org/10.1016/j. enbuild.2015.08.032.

[6] J. Kim, Y. Zhou, S. Schiavon, P. Raftery, G. Brager, Personal comfort models: predicting individuals' thermal preference using occupant heating and cooling behavior and machine learning, Build. Environ. 129 (2018) 96-106 https://doi.org/10. 1016/j.buildenv.2017.12.011.

[7] J.P. Carneiro, A. Aryal, B. Becerik-Gerber, Influencing occupant's choices by using spatiotemporal information visualization in immersive virtual environments, Build. Environ. 150 (2019) 330-338 https://doi.org/10.1016/j.buildenv.2019.01.024.

[8] W. Wang, J. Chen, T. Hong, Modeling occupancy distribution in large spaces with multi-feature classification algorithm, Build. Environ. 137 (2018) 108-117 https:// doi.org/10.1016/j.buildenv.2018.04.002.

[9] L. Ding, W. Fang, H. Luo, P.E. Love, B. Zhong, X. Ouyang, A deep hybrid learning model to detect unsafe behavior: integrating convolution neural networks and long short-term memory, Autom. ConStruct. 86 (2018) 118-124 https://doi.org/10. 1016/j.autcon.2017.11.002.

[10] C. Cruz, Semantic trajectory modeling for dynamic built environments, IEEE international conference on data science and advanced analytics (DSAA), 2017, pp. 468-476 https://doi.org/10.1109/DSAA.2017.79.

[11] M. Arslan, C. Cruz, D. Ginhac, DNAS-STriDE framework for human behavior modeling in dynamic environments, Int. Conf. Comput. Sci. (2019) 787-793 https://doi.org/10.1007/978-3-030-22750-0 79.

[12] M. Arslan, C. Cruz, D. Ginhac, Semantic trajectory insights for worker safety in dynamic environments, Autom. ConStruct. 106 (2019) 102854 https://doi.org/10. 1016/j.autcon.2019.102854.

[13] F.R. Cecconi, M. Manfren, L.C. Tagliabue, A.L.C. Ciribini, E. De Angelis, Probabilistic behavioral modeling in building performance simulation: a Monte Carlo approach, Energy Build. 148 (2017) 128-141 https://doi.org/10.1016/j. enbuild.2017.05.013.

[14] V.M. Barthelmes, Y. Heo, V. Fabi, S.P. Corgnati, Exploration of the Bayesian Network framework for modelling window control behavior, Build. Environ. 126 (2017) 318-330 https://doi.org/10.1016/j.buildenv.2017.10.011.

[15] K.R. Rozo, J. Arellana, A. Santander-Mercado, M. Jubiz-Diaz, Modelling building emergency evacuation plans considering the dynamic behaviour of pedestrians using agent-based simulation, Saf. Sci. 113 (2019) 276-284 https://doi.org/10. 1016/j.ssci.2018.11.028.

[16] P. Zhang, N. Li, Z. Jiang, D. Fang, C.J. Anumba, An agent-based modeling approach for understanding the effect of worker-management interactions on construction workers' safety-related behaviors, Autom. ConStruct. 97 (2019) 29-43 https://doi. org/10.1016/j.autcon.2018.10.015.

[17] H. Chen, X. Luo, Z. Zheng, J. Ke, A proactive workers' safety risk evaluation framework based on position and posture data fusion, Autom. ConStruct. 98 (2019) 275-288 https://doi.org/10.1016/j.autcon.2018.11.026.

[18] Y.M. Goh, C.U. Ubeynarayana, K.L.X. Wong, B.H. Guo, Factors influencing unsafe behaviors: a supervised learning approach, Accid. Anal. Prev. 118 (2018) 77-85 https://doi.org/10.1016/j.aap.2018.06.002.

[19] W. Fang, L. Ding, H. Luo, P.E. Love, Falls from heights: a computer vision-based approach for safety harness detection, Autom. ConStruct. 91 (2018) 53-61 https:// doi.org/10.1016/j.autcon.2018.02.018.

[20] L. Ding, W. Fang, H. Luo, P.E. Love, B. Zhong, X. Ouyang, A deep hybrid learning model to detect unsafe behavior: integrating convolution neural networks and long short-term memory, Autom. ConStruct. 86 (2018) 118-124 https://doi.org/10. 1016/j.autcon.2017.11.002.

[21] D. Wang, H. Li, J. Chen, Detecting and measuring construction workers' vigilance through hybrid kinematic-EEG signals, Autom. ConStruct. 100 (2019) 11-23 https://doi.org/10.1016/j.autcon.2018.12.018.

[22] H. Son, H. Choi, H. Seong, C. Kim, Detection of construction workers under varying poses and changing background in image sequences via very deep residual networks, Autom. ConStruct. 99 (2019) 27-38 https://doi.org/10.1016/j.autcon.2018. 11.033.

[23] I. Jeelani, K. Han, A. Albert, Automating and scaling personalized safety training using eye-tracking data, Autom. ConStruct. 93 (2018) 63-77 https://doi.org/10. 1016/j.autcon.2018.05.006.

[24] H. Guo, Y. Yu, Q. Ding, M. Skitmore, Image-and-skeleton-based parameterized approach to real-time identification of construction workers' unsafe behaviors, J. Constr. Eng. Manag. 144 (6) (2018) 04018042https://doi.org/10.1061/(ASCE)CO. 1943-7862.0001497.

[25] K.M. Rashid, A.H. Behzadan, Risk behavior-based trajectory prediction for construction site safety monitoring, J. Constr. Eng. Manag. 144 (2) (2018) 04017106https://doi.org/10.1061/(ASCE)CO.1943-7862.0001420.

[26] M. Zhang, T. Cao, X. Zhao, Using smartphones to detect and identify construction workers' near-miss falls based on ANN, J. Constr. Eng. Manag. 145 (1) (2018) 04018120https://doi.org/10.1061/(ASCE)CO.1943-7862.0001582.

[27] Y.K. Cho, K. Kim, S. Ma, J. Ueda, A robotic wearable exoskeleton for construction worker's safety and health, Constr. Res. Congr. (2018) 19-28 https://doi.org/10. 1061/9780784481288.003.

[28] H. Zhang, X. Yan, H. Li, R. Jin, Real-time alarming, monitoring, and locating for non-hard-hat use in construction, J. Constr. Eng. Manag. 145 (3) (2019) 04019006https://doi.org/10.1061/(ASCE)CO.1943-7862.0001629.

[29] J.M. Gómez-de-Gabriel, J.A. Fernández-Madrigal, A. López-Arquillos, J.C. RubioRomero, Monitoring harness use in construction with BLE beacons, Measurement 131 (2019) 329-340 https://doi.org/10.1016/j.measurement.2018.07.093.

[30] M. Marzouk, I. Al Daour, Planning labor evacuation for construction sites using BIM and agent-based simulation, Saf. Sci. 109 (2018) 174-185 https://doi.org/10.1016/ j.ssci.2018.04.023.

[31] A. Poulos, F. Tocornal, J.C. de la Llera, J. Mitrani-Reiser, Validation of an agentbased building evacuation model with a school drill, Transp. Res. C Emerg. Technol. 97 (2018) 82-95 https://doi.org/10.1016/j.trc.2018.10.010.

[32] S. Rai, X. Hu, Building occupancy simulation and data assimilation using a graphbased agent-oriented model, Phys. A Stat. Mech. Appl. 502 (2018) 270-287 https:/ doi.org/10.1016/j.physa.2018.02.051.

[33] A. Mahdavi, F. Tahmasebi, Predicting people's presence in buildings: an empirically based model performance analysis, Energy Build. 86 (2015) 349-355 https://doi. org/10.1016/j.enbuild.2014.10.027.

[34] K.U. Ahn, D.W. Kim, C.S. Park, P. de Wilde, Predictability of occupant presence and performance gap in building energy simulation, Appl. Energy 208 (2017) 1639-1652 https://doi.org/10.1016/j.apenergy.2017.04.083.

[35] J.A. Davis III, D.W. Nutter, Occupancy diversity factors for common university building types, Energy Build. 42 (9) (2010) 1543-1551 https://doi.org/10.1016/j enbuild.2010.03.0252010.

[36] S. Wolf, J.K. Møller, M.A. Bitsch, J. Krogstie, H. Madsen, A markov-switching model for building occupant activity estimation, Energy Build. 183 (2019) 672-683 https://doi.org/10.1016/j.enbuild.2018.11.041.

[37] R. Markovic, E. Grintal, D. Wölki, J. Frisch, C. van Treeck, Window opening model using deep learning methods, Build. Environ. 145 (2018) 319-329 https://doi.org/ 10.1016/j.buildenv.2018.09.024.

[38] Q. Huang, K. Rodriguez, N. Whetstone, S. Habel, Rapid Internet of Things (IoT) Prototype For Accurate People Counting Towards Energy Efficient Buildings, 24 ITcon, 2019, pp. 1-13 http://www.itcon.org/2019/1.

[39] A.K. Mikkilineni, J. Dong, T. Kuruganti, D. Fugate, A novel occupancy detection solution using low-power IR-FPA based wireless occupancy sensor, Energy Build. 192 (2019) 63-74 https://doi.org/10.1016/j.enbuild.2019.03.022.

[40] C.Y. Lam, W.H. Ip, An integrated logistics routing and scheduling network model with RFID-GPS data for supply chain management, Wirel. Pers. Commun. 105 (3) (2019) 803-817 https://doi.org/10.1007/s11277-019-06122-6.

[41] A. Ghosh, A. Chakraborty, D. Chakraborty, M. Saha, S. Saha, UltraSense: a nonintrusive approach for human activity identification using heterogeneous ultrasonic sensor grid for smart home environment, J. Ambient Intell. Humanized Comput. (2019) 1-22 https://doi.org/10.1007/s12652-019-01260-y.

[42] Y. Feng, M. Golparvar-Fard, Image-based localization for facilitating construction field reporting on mobile devices, Advances in Informatics and Computing in Civil and Construction Engineering, Springer, Cham, 2019, pp. 585-592 https://doi.org/ 10.1007/978-3-030-00220-6_70.

[43] G. Shtar, B. Shapira, L. Rokach, Clustering Wi-Fi fingerprints for indoor-outdoor detection, Wirel. Netw. 25 (3) (2019) 1341-1359 https://doi.org/10.1007/s11276 018-1753-9.

[44] M.U. Ali, S. Hur, Y. Park, Wi-Fi-based effortless indoor positioning system using IoT sensors, Sensors 19 (7) (2019) 1496 https://doi.org/10.3390/s19071496.

[45] J. Gong, Y. Huang, P.I. Chow, K. Fua, M.S. Gerber, B.A. Teachman, L.E. Barnes, Understanding behavioral dynamics of social anxiety among college students through smartphone sensors, Inf. Fusion 49 (2019) 57-68 https://doi.org/10.1016/ j.inffus.2018.09.002.

[46] J. Paek, J. Ko, H. Shin, A Measurement Study of BLE iBeacon and Geometric Adjustment Scheme for Indoor Location-Based Mobile Applications, Mobile Information Systems, 2016, pp. 1-13, https://doi.org/10.1155/2016/8367638 8367638

[47] Z. Riaz, M. Arslan, A.K. Kiani, S. Azhar, CoSMoS: a BIM and wireless sensor based integrated solution for worker safety in confined spaces, Autom. ConStruct. 45 (2014) 96-106 https://doi.org/10.1016/j.autcon.2014.05.010.

[48] M. Arslan, C. Cruz, D. Ginhac, Understanding worker mobility within the stay locations using HMMs on semantic Trajectories, 14th IEEE international conference on emerging technologies (ICET), 2018, pp. 1-6 https://doi.org/10.1109/ICET. 2018.8603666 . 
[49] L. Ilkovičová, P. Kajánek, A. Kopáčik, Pedestrian indoor positioning and tracking using smartphone sensors step detection and map matching algorithm, Geod. List 70 (93) (2016) 1 1-24 https://hrcak.srce.hr/156880.

[50] L. Ciabattoni, G. Foresi, A. Monteriù, L. Pepa, D.P. Pagnotta, L. Spalazzi, F. Verdini, Real time indoor localization integrating a model based pedestrian dead reckoning on smartphone and BLE beacons, J. Ambient Intell. Humanized Comput. (2017) 1-12 https://doi.org/10.1007/s12652-017-0579-0.

[51] M. Arslan, C. Cruz, D. Ginhac, Visualizing intrusions in dynamic building en vironments for worker safety, Saf. Sci. 120 (2019) 428-446.

[52] L.R. Rabiner, A tutorial on Hidden Markov Models and selected applications in speech recognition, Proc. IEEE 77 (2) (1989) 257-286 https://doi.org/10.1109/5. 18626.

[53] M. Abramowitz, I.A. Stegun, Handbook of Mathematical Functions, Dover, New York, 1972 (Chapter 6): Gamma and Related Functions, http://people.math.sfu.ca/ $\sim$ cbm/aands//abramowitz_and_stegun.pdf.

[54] R. Gatto, S.R. Jammalamadaka, The generalized von Mises distribution, Stat Methodol. 4 (3) (2007) 341-353 https://doi.org/10.1016/j.stamet.2006.11.003.

[55] W. Zucchini, An introduction to model selection, J. Math. Psychol. 44 (1) (2000) 41-61 https://doi.org/10.1006/jmps.1999.1276.

[56] T. Michelot, R. Langrock, T. Patterson, B. McClintock, E. Rexstad, Package moveHMM (Last accessed 27 July 2019),, 2019. https://cran.r-project.org/web/
packages/moveHMM/moveHMM.pdf.

[57] C. Beleites, R. Salzer, V. Sergo, Validation of soft classification models using partial class memberships: an extended concept of sensitivity \& co. applied to grading of astrocytoma tissues, Chemometr. Intell. Lab. Syst. 122 (2013) 12-22 https://doi org/10.1016/j.chemolab.2012.12.003.

[58] M. Sokolova, G. Lapalme, A systematic analysis of performance measures for classification tasks, Inf. Process. Manag. 45 (4) (2009) 427-437 https://doi.org/10. 1016/j.ipm.2009.03.002.

[59] Classification and regression training (Last accessed 27 July 2019),, 2019. https:// cran.r-project.org/web/packages/caret/caret.pdf.

[60] The Dynamo primer (Last accessed 08 Sep. 2019),, 2019. https://primer. dynamobim.org/.

[61] Occupational safety and health administration (Last accessed 08 Sep. 2019), https://www.osha.gov/laws-regs/regulations/standardnumber/1910/1910. 145AppA.

[62] R. Melfi, B. Rosenblum, B. Nordman, K. Christensen, Measuring building occupancy using existing network infrastructure, International Green Computing Conference and Workshops (2011) 1-8, https://doi.org/10.1109/IGCC.2011.6008560.

[63] B. Harbelot, H. Arenas, C. Cruz, LC3: A spatio-temporal and semantic model for knowledge discovery from geospatial datasets, Journal of Web Semantics 35 (1) (2015) 3-24, https://doi.org/10.1016/j.websem.2015.10.001. 